\title{
Probing Chirality across the Electromagnetic Spectrum with the Full Semi-classical Light-Matter Interaction
}

\author{
Martin van Horn, ${ }^{*, \dagger}$ Trond Saue, ${ }^{*, \dagger}$ and Nanna Holmgaard List ${ }^{*, \dagger}$ \\ $\dagger$ †aboratoire de Chimie et Physique Quantique, UMR 5626 CNRS - Université Toulouse \\ III-Paul Sabatier, 118 route de Narbonne, F-31062 Toulouse, France. \\ $\ddagger$ School of Engineering Sciences in Chemistry, Biotechnology and Health (CBH), \\ Department of Theoretical Chemistry and Biology, KTH Royal Institute of Technology, \\ Stockholm, Sweden. \\ E-mail: mvanhorn@irsamc.ups-tlse.fr; trond.saue@irsamc.ups-tlse.fr; nalist@kth.se
}

\begin{abstract}
We present a formulation and implementation of anisotropic and isotropic electronic circular dichroism (ECD) using the full semi-classical light-matter interaction operator within a four-component relativistic framework. The account of both beyond-first-order light-matter interactions and relativistic effects allows us to investigate the ECD response across the electromagnetic spectrum from optical to X-ray wavelengths where relativistic selection rules and spatial field variations gain increasing importance. We consider the isotropic and oriented ECD across the valence, sulfur $L$ - and $K$-edge transitions in the simplest disulfides, $\mathrm{H}_{2} \mathrm{~S}_{2}$ and $\left(\mathrm{CH}_{3} \mathrm{~S}\right)_{2}$, and evaluate the influence of the full interaction by comparing to a traditional truncated formulation in the Coulomb gauge (velocity representation). Additionally, we demonstrate that in the relativistic formalism it is possible to work in the velocity representation, hence keeping order-by-order
\end{abstract}


gauge-origin invariance, contrary to multipolar gauge, yet being able to distinguish electric and magnetic multipole contributions. Going beyond a first-order treatment in the wave vector is mandatory in the higher-energy end of the soft X-ray region where the consequent intensity redistribution becomes significant. While the sulfur $K$-edge absorption spectrum is essentially unaffected by this redistribution, the signed differential counterpart is not: at least third-order contributions are required to describe the differential absorption profile that is otherwise overestimated by a factor of about two. 


\section{Introduction}

Chirality is fundamental to life on Earth, and the origin of homochirality of natural amino acids and sugars remains an unresolved question. ${ }^{1}$ Enantiomers of a chiral molecule interact differently with chiral objects, such as chiral receptors or left and right circularly polarized light. The former is central in chiral molecular recognition, e.g. drug-receptor binding, while the latter enables spectroscopic detection of chirality. Electronic circular dichroism (ECD) is the lowest-order natural chiroptical response: it measures the differential linear absorption between left and right circularly polarized light in the spectral region of electronic transitions. ${ }^{2}$ Many biomolecules are optically active either intrinsically due to the presence of a chiral center or induced through structural and electronic perturbations by a chiral environment such as through electronic coupling within a chiral arrangement of achiral chromophores. ${ }^{3,4}$ ECD in the valence region is therefore extensively used to provide stereochemical, conformational and binding information of molecular systems. ${ }^{5}$ Anisotropic circular dichroism allows for additional information which would otherwise be lost in the isotropic signal. As an example, anisotropic ECD was recently proposed as a way to distinguish different transitions in light-harvesting complex II oriented by its embedding in a membrane. ${ }^{6,7}$

While the discovery of natural optical activity in the visible region dates back more than two hundred years to the work of $\operatorname{Arago}^{8}$ and Biot, ${ }^{9}$ its existence in the X-ray range was only established in 1998: the detection of X-ray natural circular dichroism (XNCD) in non-centrosymmetric crystals, ${ }^{10-13}$ was enabled by the advent of intense third-generation synchrotron X-ray sources with full polarization control. Like other resonant core-level spectroscopies, XNCD exploits the site- and element-specificity of X-ray probes to provide in-

formation about the local symmetry around the absorbing atom(s). In addition to chiral crystals, XNCD has also been measured for small organic molecules including several amino acids (see e.g. Refs. 14-17).

From a multipole expansion of the light-matter interactions in orders of the wave vec- 
tor, the leading-order contributions to ECD (i.e., first order) are governed by the electricdipole/magnetic-dipole (E1-M1) and electric-dipole/electric-quadrupole (E1-E2) interference terms. The latter is traceless and hence vanishes for isotropic samples. While the electric-dipole/magnetic-dipole interference dominates in the optical region (in the centerof-mass frame), it is difficult to observe at $K\left(L_{1}\right)$-edges, ${ }^{18,19}$ because the magnetic transitiondipole moment involving the atomic-like 1(2)s-orbitals becomes forbidden within a zerothorder approximation in the non-relativistic domain. ${ }^{13,20}$ The electric-dipole/electric-quadrupole interference term, on the other hand, gains intensity in the X-ray region, due to its dependency on the spatial variations of the electromagnetic field. ${ }^{20,21}$ For these reasons, substantial XNCD signals are expected only for oriented samples, although weak electricdipole/magnetic-dipole contributions have been reported in the absence of orientational order (e.g., in solution or powdered samples ${ }^{19,22}$ ).

Theoretical simulations play a key role in the assignment of experimental ECD spectra. Traditionally these rely on the aforementioned multipole expansion of the ECD signal through first order in the wave vector. However, caution has to be exercised to ensure gaugeorigin independent results, e.g. by performing calculations in the velocity representation ${ }^{23}$ or by employing gauge-including atomic orbitals. ${ }^{24,25}$ An additional aspect arising in the X-ray regime, where the wavelength of the electromagnetic field approaches molecular dimensions, is the importance of higher-order terms in the expansion. To address these issues in the context of linear absorption of linearly polarized light, we recently proposed using the full semi-classical light-matter interaction operator in both the non-relativistic ${ }^{26,27}$ and relativistic regimes. ${ }^{28}$ In addition to providing compatible transformation properties for both light and matter, the relativistic formulation is particularly simple because the light-matter interaction is linear in both the scalar and vector potentials.

In this work, we present a four-component relativistic formulation and implementation of isotropic and anisotropic ECD using the full semi-classical light-matter interaction. This allows us to investigate the ECD response across the electromagnetic spectrum, from optical to 
X-ray regimes. So far, previous theoretical studies of the isotropic XNCD of molecules have only considered the first-order truncated interaction in a non-relativistic framework. ${ }^{18,22,29-35}$ In these studies, the only source of non-vanishing response at the $L_{1^{-}}$and $K$-edges is the polarization away from atomic symmetry of the core orbitals. Our implementation accounts for two additional possible contributions: $(i)$ effects of beyond first-order light-matter interactions and (ii) inclusion of relativistic effects, notably spin-orbit coupling that modifies selection rules (in particular, the magnetic transition-dipole selection rule ${ }^{36,37}$ ). Consequently, this allows, for the first time, to realistically examine the ECD response of molecules across the valence and X-ray regimes.

As test systems, we consider the simplest disulfide chromophore models, dihydrogen disulfide $\mathrm{H}_{2} \mathrm{~S}_{2}$ and dimethyl disulfide $\left(\mathrm{CH}_{3} \mathrm{~S}\right)_{2}$. Because of the low disulfide torsional barriers $\left(\sim 6-11 \mathrm{kcal} / \mathrm{mol}^{38-41}\right)$, the two enantiomeric forms $(P$ - and $M$-helix) cannot be resolved experimentally. However, the disulfide bridge is an important structural element in proteins, ${ }^{42}$ where it preferentially occurs in non-planar, chiral conformations $\left(C_{2}\right.$ symmetry) and hence displays structurally-induced axial chirality. An interesting perspective for complex systems (e.g., proteins) is the potential use of XNCD as a local probe of chirality. ${ }^{30,34}$ This could potentially complement the delocalized conformational information encoded in valence ECD. Because of its computational tractability, $\mathrm{H}_{2} \mathrm{~S}_{2}$ has been widely used to benchmark electronic structure methods for the calculation of chiroptical properties. ${ }^{43-49}$ For the same reason, Goulon et al. also used it to estimate relative magnitudes of XNCD responses within the first-order truncated interaction and non-relativistic framework, reporting values below the experimental detection limits. ${ }^{18}$ Here, we revisit the ECD of the disulfide chromophore across the valence, $L-$ and $K$-edges, going beyond these approximations. 


\section{Theory}

\subsection{Full light-matter interaction}

We begin with a description of our ECD formulation using the full semi-classical light-matter interaction, which has been implemented in a development version of the DIRAC electronic structure program. ${ }^{50,51}$ We consider a circularly polarized monochromatic (angular frequency $\omega)$ electromagnetic field with electric and magnetic components given by

$$
\begin{aligned}
& \mathbf{E}^{(M)}(\mathbf{r}, t)=\frac{1}{\sqrt{2}} E_{\omega}\left[\boldsymbol{\epsilon}_{1} \sin (\mathbf{k} \cdot \mathbf{r}-\omega t)+(-1)^{M} \boldsymbol{\epsilon}_{2} \cos (\mathbf{k} \cdot \mathbf{r}-\omega t)\right] \\
& \mathbf{B}^{(M)}(\mathbf{r}, t)=\frac{1}{\sqrt{2}} \frac{E_{\omega}}{c}\left[\boldsymbol{\epsilon}_{2} \sin (\mathbf{k} \cdot \mathbf{r}-\omega t)-(-1)^{M} \boldsymbol{\epsilon}_{1} \cos (\mathbf{k} \cdot \mathbf{r}-\omega t)\right],
\end{aligned}
$$

where appears the polarization vectors $\boldsymbol{\epsilon}_{1}$ and $\boldsymbol{\epsilon}_{2}$ that, together with the wave vector $\mathbf{k}=k \mathbf{e}_{k}$

$\left(k=\frac{\omega}{c}\right)$, form a right-handed coordinate system; the field amplitude $E_{\omega}$, and the integer $M$ that specifies the handedness of the field, i.e., left (even) and right (odd) circularly polarized light, respectively, following the IUPAC recommendation. ${ }^{52}$ Such an electromagnetic wave is conventionally represented in the Coulomb gauge by the scalar and vector potentials

$$
\begin{aligned}
\phi^{(M)}(\mathbf{r}, t) & =0 \\
\mathbf{A}^{(M)}(\mathbf{r}, t) & =-\frac{1}{\sqrt{2}} \frac{E_{\omega}}{\omega}\left[\boldsymbol{\epsilon}_{1} \cos (\mathbf{k} \cdot \mathbf{r}-\omega t)-(-1)^{M} \boldsymbol{\epsilon}_{2} \sin (\mathbf{k} \cdot \mathbf{r}-\omega t)\right] .
\end{aligned}
$$

In the relativistic domain, this leads to an interaction operator for left/right-handed (L/R) circularly polarized light of the form

$$
\hat{V}_{\mathrm{L} / \mathrm{R}}(t)=e c\left(\boldsymbol{\alpha} \cdot \mathbf{A}_{\mathrm{L} / \mathrm{R}}\right)=-\frac{1}{2} E_{\omega} \sum_{ \pm \omega} \hat{T}_{\mathrm{L} / \mathrm{R}}(\omega) e^{-i \omega t} ; \quad \hat{T}_{\mathrm{L} / \mathrm{R}}(\omega)=\frac{e}{\omega}\left(c \boldsymbol{\alpha} \cdot \boldsymbol{\epsilon}_{\mathrm{L} / \mathrm{R}}\right) e^{i \mathbf{k} \cdot \mathbf{r}}
$$


where $\hat{T}_{\mathrm{L} / \mathrm{R}}(\omega)$ is the effective interaction operator (cf. Ref. 28), $\boldsymbol{\alpha}$ are Dirac matrices and

$$
\boldsymbol{\epsilon}_{\mathrm{L} / \mathrm{R}}=\frac{1}{\sqrt{2}}\left(\boldsymbol{\epsilon}_{1} \pm i \boldsymbol{\epsilon}_{2}\right)=\boldsymbol{\epsilon}_{\mathrm{R} / \mathrm{L}}^{*}
$$

For notational simplicity, we tacitly assume the summation over electrons in the effective interaction operator in Eq. (3). Considerable simplifications arise from the fact that the relativistic interaction operator is linear in the vector potential and there is no separation into spin and spatial parts.

The linear absorption cross section between initial and final states, $|i\rangle$ and $|f\rangle$, respectively, is then given by

$$
\sigma_{\mathrm{L} / \mathrm{R}}(\omega)=\frac{\pi \omega}{\varepsilon_{0} \hbar c}\left|\boldsymbol{\epsilon}_{\mathrm{L} / \mathrm{R}} \cdot \mathbf{T}\right|^{2} f\left(\omega, \omega_{f i}, \gamma_{f i}\right) ; \quad \mathbf{T}=\left\langle f\left|\frac{e}{\omega} c \boldsymbol{\alpha} e^{i \mathbf{k} \cdot \mathbf{r}}\right| i\right\rangle .
$$

The use of Fermi's golden rule for a transition between discrete states is justified by the inclusion of a (Lorentzian) lineshape function $f\left(\omega, \omega_{f i}, \gamma_{f i}\right)$ expressing the finite lifetime of excited states (see discussion in Ref. 28). Moreover, the lineshape function, having the dimension of time, has to be included in order for the absorption cross section to have the correct dimension of area. On the other hand, the dimensionless differential oscillator strength is obtained from the absorption cross section by the pre-factor substitution $\frac{\pi \omega}{\epsilon_{0} \hbar c} \rightarrow$ $\frac{2 m_{e} \omega}{\hbar e^{2}}$ and removal of the lineshape function. In the interest of compact expressions we shall in the following carry out our derivations using oscillator strengths rather than absorption cross sections.

Evaluating the differential oscillator strengths between left and right circularly polarized light starting from Eq. (5) provides the expression for the anisotropic differential oscillator 
strength using the full semi-classical interaction operator

$$
\begin{aligned}
\Delta f=f_{\mathrm{L}}-f_{\mathrm{R}} & =\frac{2 m_{e} \omega}{\hbar e^{2}}\left\{\left(\boldsymbol{\epsilon}_{\mathrm{L}} \cdot \mathbf{T}\right)\left(\boldsymbol{\epsilon}_{\mathrm{R}} \cdot \mathbf{T}^{*}\right)-\left(\boldsymbol{\epsilon}_{\mathrm{L}} \cdot \mathbf{T}^{*}\right)\left(\boldsymbol{\epsilon}_{\mathrm{R}} \cdot \mathbf{T}\right)\right\} \\
& =\frac{2 m_{e} \omega}{\hbar e^{2}}\left(\boldsymbol{\epsilon}_{\mathrm{L}} \times \boldsymbol{\epsilon}_{\mathrm{R}}\right) \cdot\left(\mathbf{T} \times \mathbf{T}^{*}\right)=-i \frac{2 m_{e} \omega}{\hbar e^{2}} \mathbf{e}_{k} \cdot\left(\mathbf{T} \times \mathbf{T}^{*}\right)
\end{aligned}
$$

where we have used Eq. (4) and the vector relation

$$
(\mathbf{a} \times \mathbf{b}) \cdot(\mathbf{c} \times \mathbf{d})=(\mathbf{a} \cdot \mathbf{c})(\mathbf{b} \cdot \mathbf{d})-(\mathbf{a} \cdot \mathbf{d})(\mathbf{b} \cdot \mathbf{c}) .
$$

This final, compact expression represents the relativistic extension of the expression previously reported by Hansen and Avery. ${ }^{53}$

It is instructive to compare the differential oscillator strength in Eq. (6) (ECD) with the oscillator strength associated with linearly polarized light. The latter is obtained by replacing the complex polarization vector $\boldsymbol{\epsilon}_{\mathrm{L} / \mathrm{R}}$ in Eq. (5) by the polarization vector $\boldsymbol{\epsilon}$ giving the direction of the electric field vector in the plane perpendicular to the wave vector. One immediately notes that the differential oscillator strength contains no reference to polarization vectors at all, which can be understood as a manifestation of axial symmetry. This simplifies the rotational averaging appropriate for samples such as liquids and gases, with freely tumbling molecules. Specificially, the rotational average involves three angles $(\theta, \phi, \chi)$ : the first two are associated with an average over propagation direction, whereas the polarization angle $\chi$ now becomes redundant.

\subsection{Truncated interaction}

\subsubsection{Multipoles in the velocity representation}

In our previous work on the absorption of linearly polarized light, we considered two schemes involving truncated light--matter interaction. ${ }^{28}$ In the first scheme, referred to as the generalized velocity representation, the truncated interaction is obtained via a Taylor expansion 
of the oscillator strength in orders of the wave vector. Applying this to Eq. (6), we obtain the following expressions for the differential oscillator strength to even and odd order in the wave vector

$$
\begin{aligned}
\Delta f^{[2 n]} & =\frac{2 m_{e} \omega}{\hbar e^{2}} \mathbf{e}_{k} \cdot \sum_{m=0}^{n}(-1)^{m}\left(2-\delta_{m 0}\right) \operatorname{Im}\left\{\left(\mathbf{T}^{[n+m]} \times \mathbf{T}^{[n-m] *}\right)\right\}=0 \\
\Delta f^{[2 n+1]} & =\frac{2 m_{e} \omega}{\hbar e^{2}} \mathbf{e}_{k} \cdot \sum_{m=0}^{n}(-1)^{m} 2 \operatorname{Re}\left\{\mathbf{T}^{[n+m+1]} \times \mathbf{T}^{[n-m] *}\right\}
\end{aligned}
$$

where

$$
\mathbf{T}^{[n]} \cdot \boldsymbol{\epsilon}_{\mathrm{L} / \mathrm{R}}=\left\langle f\left|\hat{T}_{\mathrm{L} / \mathrm{R}}^{[n]}\right| i\right\rangle ; \quad \hat{T}_{\mathrm{L} / \mathrm{R}}^{[n]}=\frac{e}{\omega} \frac{1}{n !}\left(i c \boldsymbol{\alpha} \cdot \boldsymbol{\epsilon}_{\mathrm{L} / \mathrm{R}}\right)(\mathbf{k} \cdot \mathbf{r})^{n}
$$

Note that only odd-order terms are sensitive to the handedness and hence contribute to the expansion. This is a consequence of the time-reversal symmetry of the truncated interaction operators, making the corresponding transition moments real. ${ }^{28}$

In the second scheme, referred to as generalized length representation, we transform to multipolar gauge (mg). ${ }^{28,54-58}$ Using the Einstein summation convention, the full interaction operator is then expressed as

$\hat{V}_{\mathrm{mg}}(\omega)=\sum_{n=0}^{\infty} \hat{V}_{\mathrm{mg}}^{[n]}(\omega) ;\left\{\begin{array}{l}\hat{V}_{\mathrm{mg}}^{[0]}(\omega)=-\hat{Q}_{p}^{[1]} E_{p}^{[0]} \\ \hat{V}_{\mathrm{mg}}^{[n \neq 0]}(\omega)=-\frac{1}{(n+1) !} \hat{Q}_{j_{1} \ldots j_{n} p}^{[n+1]} E_{p ; j_{1} \ldots j_{n}}^{[n]}-\frac{1}{n !} \hat{m}_{j_{1} \ldots j_{n-1} ; r}^{[n]} B_{r ; j_{1} \ldots j_{n-1}}^{[n-1]},\end{array}\right.$,

where appears electric and magnetic multipole operators

$$
\begin{aligned}
\hat{Q}_{j_{1} \ldots j_{n} p}^{[n+1]} & =-e r_{j_{1}} r_{j_{2}} \ldots r_{j_{n}} r_{p} \\
\hat{m}_{j_{1} \ldots j_{n-1} ; r}^{[n]} & =\frac{n}{n+1} r_{j_{1}} r_{j_{2}} \ldots r_{j_{n-1}}(\mathbf{r} \times \hat{\mathbf{j}})_{r} ; \quad \hat{\mathbf{j}}=-e c \boldsymbol{\alpha}
\end{aligned}
$$

that couple to the electric and magnetic fields and their derivatives

$$
F_{p ; j_{1} \ldots j_{n}}^{[n]}=\left.\frac{\partial^{n} F_{p}}{\partial r_{j_{1}} \partial r_{j_{2}} \ldots \partial r_{j_{n}}}\right|_{\mathbf{r}=\mathbf{0}} ; \quad \mathbf{F}=\mathbf{E}, \mathbf{B}
$$


at a selected expansion point, here set to the origin. The corresponding $n$ th-order effective interaction operator is given by

$\hat{T}_{\mathrm{mg} ; \mathrm{L} / \mathrm{R}}^{[n]}(\omega)=-e\left[\frac{1}{(n+1) !}\left(\mathbf{r} \cdot \boldsymbol{\epsilon}_{\mathrm{L} / \mathrm{R}}\right)(i \mathbf{k} \cdot \mathbf{r})^{n}-\frac{i}{\omega} \frac{n}{(n+1) !}\left(i \mathbf{k} \times \boldsymbol{\epsilon}_{\mathrm{L} / \mathrm{R}}\right) \cdot(\mathbf{r} \times c \boldsymbol{\alpha})(i \mathbf{k} \cdot \mathbf{r})^{n-1}\right]$.

On component form, the effective interaction operator is conveniently expressed as

$$
\hat{T}_{\mathrm{mg}}^{[n]}(\omega)=i^{n} \epsilon_{\mathrm{L} / \mathrm{R} p} k_{j_{1}} k_{j_{2}} \ldots k_{j_{n}} \hat{X}_{j_{1} \ldots j_{n} ; p}^{[n]}(\omega)
$$

in terms of a multipole operator ${ }^{28}$

$$
\hat{X}_{j_{1} \ldots j_{n} ; p}^{[n]}(\omega)=\frac{1}{(n+1) !} \hat{Q}_{j_{1} \ldots j_{n}, p}^{[n+1]}-\frac{i}{\omega} \frac{1}{n !} \hat{m}_{j_{1 \ldots j} \ldots j_{n-1} ; r}^{[n]} \varepsilon_{r j_{n} p}
$$

$\left(\varepsilon_{r j_{n} p}\right.$ is the Levi-Civita symbol). However, in contrast to the generalized velocity representation, we now loose origin independence of oscillator strengths. ${ }^{28}$

In our previous work, ${ }^{28}$ however, we have have shown that it is possible within the generalized velocity representation to distinguish electric and magnetic multipole components, while maintaining origin independence of the oscillator strengths, although its individual components still exhibit this dependency. The operator manipulations required for this separation do not involve commutators with the Hamiltonian and are therefore also valid when using finite basis sets. We here review and expand this result. Using the vector relation in Eq. (7), the effective interaction operator of Eq. (10) can be rewritten as

$$
\begin{aligned}
\hat{T}_{\mathrm{L} / \mathrm{R}}^{[n]} & =\frac{e}{\omega}\left[\frac{1}{(n+1) !}\left\{\left(i c \boldsymbol{\alpha} \cdot \boldsymbol{\epsilon}_{\mathrm{L} / \mathrm{R}}\right)(\mathbf{k} \cdot \mathbf{r})^{n}+n(\mathbf{k} \cdot i c \boldsymbol{\alpha})\left(\boldsymbol{\epsilon}_{\mathrm{L} / \mathrm{R}} \cdot \mathbf{r}\right)(\mathbf{k} \cdot \mathbf{r})^{n-1}\right\}\right. \\
& \left.+\frac{n}{(n+1) !}\left(\mathbf{k} \times \boldsymbol{\epsilon}_{\mathrm{L} / \mathrm{R}}\right) \cdot(\mathbf{r} \times i c \boldsymbol{\alpha})(\mathbf{k} \cdot \mathbf{r})^{n-1}\right],
\end{aligned}
$$

As evident upon comparison to Eq. (15), we have effectively recovered the magnetic multipole part of the operator, and, in fact, writing the corresponding nth-order interaction 
operator $\hat{V}_{\mathrm{L} / \mathrm{R}}^{[n]}$ out on component form, we recover the expression of Eq. (11), with the important modification that the electric multipole operator in the length represention Eq. (12) is replaced by its counterpart in the velocity representation

$$
\hat{\mathcal{Q}}_{j_{1} \ldots j_{n} p}^{n+1]}=\frac{i e}{\omega} r_{j_{1}} \ldots r_{j_{n-1}}\left(\left(c \alpha_{p}\right) r_{j_{n}}+n\left(c \alpha_{j_{n}}\right) r_{p}\right)
$$

This somewhat curious form is perhaps best understood by noting that the operator can equally well be expressed as

$$
\mathcal{Q}_{j_{1} \ldots j_{n} p}^{[n+1]}=\frac{i e}{\omega} \underbrace{\left(c \alpha_{j_{1}} r_{j_{2}} \ldots r_{j_{n}} r_{p}+\ldots+r_{j_{1}} r_{j_{2}} \ldots c \alpha_{j_{n}} r_{p}+r_{j_{1}} r_{j_{2}} \ldots r_{j_{n}} c \alpha_{p}\right)}_{(n+1) \text { terms }}
$$

being perfectly symmetric in all indices $\left\{j_{1} \ldots j_{n} p\right\}$, just as Eq. (12). The more compact expression in Eq. (19) is possible because the relativistic velocity operator co commutes with the coordinates, contrary to the corresponding non-relativistic operator $\mathbf{p} / m$. Finally, using the commutator relation with the Hamiltonian

$$
-\frac{i}{\hbar}\left[\left(\mathbf{r} \cdot \boldsymbol{\epsilon}_{\mathrm{L} / \mathrm{R}}\right)(\mathbf{k} \cdot \mathbf{r})^{n}, \hat{h}\right]=\left(c \boldsymbol{\alpha} \cdot \boldsymbol{\epsilon}_{\mathrm{L} / \mathrm{R}}\right)(\mathbf{k} \cdot \mathbf{r})^{n}+n\left(\mathbf{r} \cdot \boldsymbol{\epsilon}_{\mathrm{L} / \mathrm{R}}\right)(\mathbf{k} \cdot c \boldsymbol{\alpha})(\mathbf{k} \cdot \mathbf{r})^{n-1},
$$

one can show that transition moments of the electric multipole operator in the length and velocity representation will be identical. ${ }^{28}$ However, very importantly, this generally only holds in a complete basis.

The odd-order oscillator strength of Eq. (9) may now be expressed as

$$
\Delta f^{[2 n+1]}=\frac{2 m_{e} \omega}{\hbar e^{2}} k^{2 n+1} e_{k ; j_{1}} \ldots e_{k ; j_{2 n+1}} \mathbf{e}_{k} \cdot \sum_{m=0}^{n}(-1)^{m} 2\left(\mathbf{X}_{j_{1} \ldots j_{n+m}+1}^{[n+m+1]} \times \mathbf{X}_{j_{n+m+2} \ldots j_{2 n+1}}^{[n-m]}\right)
$$

where components of the real vector $\mathbf{X}_{j_{1} \ldots j_{n}}^{[n]}$ are given as the transition moments of the multipole operator in Eq. (17) in the velocity representation. 


\subsubsection{Angular dependence of truncated ECD}

In the anisotropic case both the full and truncated differential oscillator strengths (Eqs. (6) and (22), respectively) are functions of the orientation of the incident radiation. In the case of truncated interaction we make this angular dependence explicit by writing the unit wave vector as

$$
\mathbf{e}_{k}=(\sin \theta \cos \phi, \sin \theta \sin \phi, \cos \theta)
$$

The first-order differential oscillator strength can then be expanded as

$$
\begin{aligned}
\Delta f^{[1]}(\theta, \phi) & =e_{k ; i} R_{i j}^{[1]} e_{k ; j} \\
& =R_{\text {iso }}^{[1]}+\frac{1}{2}\left(R_{z z}^{[1]}-R_{\text {iso }}^{[1]}\right)\left(3 \cos ^{2} \theta-1\right) \\
& +\frac{1}{2}\left(R_{x z}^{[1]}+R_{z x}^{[1]}\right) \sin 2 \theta \cos \phi+\frac{1}{2}\left(R_{y z}^{[1]}+R_{z y}^{[1]}\right) \sin 2 \theta \sin \phi \\
& +\frac{1}{2}\left(R_{x x}^{[1]}-R_{y y}^{[1]}\right) \sin ^{2} \theta \cos 2 \phi+\frac{1}{2}\left(R_{x y}^{[1]}+R_{y x}^{[1]}\right) \sin ^{2} \theta \sin 2 \phi
\end{aligned}
$$

where we have introduced the rotational strength tensor $R_{i j}^{[1]}$ (cf. Ref. 59) and its isotropic part $R_{\text {iso }}^{[1]}$, known as the rotational strength

$$
R_{i j}^{[1]}=\frac{4 m_{e} \omega^{2}}{\hbar c e^{2}}\left(\mathbf{X}_{j}^{[1]} \times \mathbf{X}^{[0]}\right)_{i} ; \quad R_{\mathrm{iso}}^{[1]}=\frac{1}{3}\left(R_{x x}^{[1]}+R_{y y}^{[1]}+R_{z z}^{[1]}\right)
$$

From Eq. (24) we see that the anisotropic part of $\Delta f^{[1]}(\theta, \phi)$ is a linear combination of $d$ orbitals weighted by the relevant elements of the rotatory strength tensor. This can be made more explicit by rewriting the first-order differential oscillator strength as

$$
\begin{aligned}
\Delta f^{[1]}(\theta, \phi) & =\sqrt{15} R_{\mathrm{iso}}^{[1]} s+\sqrt{3}\left(R_{z z}^{[1]}-R_{\mathrm{iso}}^{[1]}\right) d_{z^{2}}+\left(R_{x x}^{[1]}-R_{y y}^{[1]}\right) d_{x^{2}-y^{2}} \\
& +\left(R_{x z}^{[1]}+R_{z x}^{[1]}\right) d_{x z}+\left(R_{y z}^{[1]}+R_{z y}^{[1]}\right) d_{y z}+\left(R_{x y}^{[1]}+R_{y x}^{[1]}\right) d_{x y}
\end{aligned}
$$

where we have used a common normalization for all solid harmonics.

From Eq. (22) we see that rotational strength tensors can be generalized to arbitrary 
odd orders. It will also be seen that the differential oscillator strength $\Delta f^{[2 n+1]}$ contains products of $2(n+1)$ components of the unit wave vector and is therefore spanned by spherical harmonics of even $\ell=0,2, \ldots 2(n+1)$.

In the Supplementary Information (SI) the (first-order) rotational strength tensor is analyzed in terms of symmetry for the $C_{2}$ point group of the disulfide configurations considered, assuming the rotation axis to coincide with the $z$-axis. We also show that that the rotational strength tensor in the generalized velocity representation is invariant under a shift of origin, also in a finite basis, which generally does not hold true in the generalized length representation. As pointed out above, the separation into electric and magnetic multipole contributions generally depends on the choice of gauge origin, and thus, is not unique. However, the presence of symmetry may introduce additional gauge-origin invariances. ${ }^{60}$ The E1-M1 and E1-E2 contributions to the $R_{z *}^{[1]}$ elements are separately invariant under origin shifts along the rotation axes (Section S2); in $A$ symmery this holds for all elements of the full rotational strength tensor.

In the case of the full interaction, the factorization of radial and angular parts is possible using the plane wave expansion

$$
e^{i \mathbf{k} \cdot \mathbf{r}}=4 \pi \sum_{\ell=0}^{\infty} \sum_{m=-\ell}^{\ell} i^{\ell} j_{\ell}(k r) Y_{\ell}^{m}\left(\mathbf{e}_{k}\right) Y_{\ell}^{m *}\left(\mathbf{e}_{r}\right)
$$

in terms of spherical harmonics $Y_{\ell}^{m}$ and spherical Bessel functions $j_{\ell}$. However, it is more cumbersome since it involves infinite sums over angular momentum $\ell$ and will not be pursued further here.

\subsection{Rotational average}

As pointed out above, rotational averaging is simplified for the differential oscillator strength because of the absence of reference to the polarization vectors. It is therefore limited to the 
solid angle, indicating the propagation direction, and is defined as

$$
\langle g(\mathbf{r})\rangle_{\theta, \phi}=\frac{1}{4 \pi} \int_{0}^{2 \pi} \int_{0}^{\pi} g(\mathbf{r}) \sin \theta d \theta d \phi
$$

In the case of the full light-matter interaction, the rotational average is handled numerically using a Lebedev grid. ${ }^{27,28}$ In the case of the truncated interaction, the rotational average is expressed as

$\left\langle\Delta f^{[2 n+1]}\right\rangle_{\theta, \phi}=\Delta f_{i s o}^{[2 n+1]}=\frac{2 m_{e} \omega}{\hbar e^{2}} k^{2 n+1}\left\langle e_{k ; j_{1}} \ldots e_{k ; j_{2 n+1}} \mathbf{e}_{k}\right\rangle_{\theta, \phi} \cdot \sum_{m=0}^{n}(-1)^{m} 2\left\{\mathbf{X}_{j_{1} \ldots j_{n+m+1}}^{[n+m+1]} \times \mathbf{X}_{j_{n+m+2} \ldots j_{2 n}}^{[n-m]}\right\}$

To lowest order the isotropic differential oscillator strength becomes

$$
\left\langle\Delta f^{[1]}\right\rangle_{\theta, \phi}=\frac{4 m_{e} \omega^{2}}{3 \hbar c e^{2}} \sum_{j}\left(\mathbf{X}_{j}^{[1]} \times \mathbf{X}^{[0]}\right)_{j}=\frac{8 m_{e} \omega^{2}}{3 \hbar c e^{2}} \sum_{j}\left\langle f\left|\hat{\mathcal{Q}}_{j}^{[1]}\right| i\right\rangle\left\langle f\left|-\frac{i}{\omega} \hat{m}_{j}^{[1]}\right| i\right\rangle=R_{\text {iso }}^{[1]}
$$

where we have used that $\left\langle e_{k ; j} \mathbf{e}_{k}\right\rangle_{\theta, \phi}=\frac{1}{3} \mathbf{e}_{j}$ and that the E1-E2 contribution vanishes since its elements form a traceless matrix.

\section{$3 \quad$ Implementation Details}

In practice, the interaction operator is separated into Hermitian and anti-Hermitian parts for compatibility with the quaternion symmetry scheme of DIRAC (see Refs. 28,61). The im-

plementation of Eq. (6) requires the same integrals as the linear oscillator strength, and thus our previous integral implementation could be readily extended to ECD. ${ }^{26,27}$ The integral evaluation over Lebedev points has been parallelized using MPI (Message Passing Interface). 


\section{Computational Details}

The geometries of $\mathrm{H}_{2} \mathrm{~S}_{2}$ and $\left(\mathrm{CH}_{3} \mathrm{~S}\right)_{2}$ were obtained using the B3LYP ${ }^{62-65}$ exchange-correlation functional and the cc-pVTZ ${ }^{66,67}$ basis set. Geometry optimizations were performed in Gaussian 16. ${ }^{68}$ To mimic the $\chi_{3}$ disulfide angle typical for protein structures, ${ }^{69}$ we performed constrained geometry optimizations for $\chi_{3}=-87^{\circ}$, corresponding to $M$-helical chirality. ${ }^{70}$ The restricted excitation window approach ${ }^{71,72}$ was used to selectively target the sulfur $L$ and $K$-edges. This also eliminates the issue of artificial transitions to quasi-continuum orbitals caused by finite basis set effects that otherwise often interferes simulations at the $L$-edge. ${ }^{73,74}$ A Gaussian model was employed for the nuclear charge distribution, ${ }^{75}$ and an 86-point Lebedev grid $\left(L_{\max }=12\right)$ was used for the isotropic averaging of the differential linear absorption based on the full interaction operator. The gauge origin was placed in the center-of-mass (COM) and spatial symmetry was invoked in all cases except for the gauge-origin dependence calculations.

Excitation energies, linear and differential absorption cross sections for the full interaction operator as well as the multipole expansions within the generalized velocity gauge were computed using the PBE0 ${ }^{76,77}$ exchange-correlation functional and the uncontracted aug-pcX-3 ${ }^{78}$ and aug-pc-3 $3^{79-81}$ basis sets for sulfur and hydrogen, respectively. The pcX-n basis set series was developed for describing core-excitation processes using the $\triangle \mathrm{SCF}$ (SelfConsistent Field) approach at both the nonrelativistic and relativistic levels. The small component basis sets were generated within the condition of restricted kinetic balance. The relativistic calculations were performed using a Dirac-Coulomb Hamiltonian in which the (SS|SS) integrals are replaced by an interatomic SS energy correction. ${ }^{82}$ Gauge-origin invariance of our implementation of the full semi-classical formulation of the isotropic and anisotropic rotatory strengths (Eq. (6)) and its first-order truncated counterpart was confirmed numerically by shifting the gauge-origin (from 0 to $100 a_{0}$ ) along the $C_{2}$ axis. This leads to a redistribution of the E1-E2 and E1-M1 contributions to $R_{x x}^{[1]}$ and $R_{y y}^{[1]}$ for transitions of $B$ symmetry. As expected, the results remained unchanged for both the full and 
truncated formulations (data not shown). Simulated spectra were obtained by convolving the stick spectrum with Gaussian lineshape functions with full width at half maximum (FWHM) of $0.4 \mathrm{eV}$, and those for $\left(\mathrm{CH}_{3} \mathrm{~S}\right)_{2}$ were shifted by different offsets for each absorption edge so as to match their experimental counterparts.

\section{Results and Discussion}

Before considering the ECD response of the disulfides, we assign the linear absorption features across the valence and X-ray regions. We initially focus on $\left(\mathrm{CH}_{3} \mathrm{~S}\right)_{2}$ for which experimental gas-phase absorption spectra are available. ${ }^{83-87}$ Expectedly, and as shown in Figure S1, the spectral profiles for $\mathrm{H}_{2} \mathrm{~S}_{2}$ are similar, and because of its greater computational tractability, we consider this minimal disulfide in subsequent analyses.

Figure 1 displays the rotationally averaged linear and differential absorption spectra for valence, sulfur $L$ - and $K$-edge transitions of $\left(\mathrm{CH}_{3} \mathrm{~S}\right)_{2}$, computed using the full interaction operator (green shading) with corresponding oscillator strengths indicated as green sticks. Hereafter, we explicitly indicate the results of the full interaction with the superscript "full". For comparison, we also provide the lowest non-vanishing terms in the truncated generalized velocity representation (orange lines) for linear and differential absorption, i.e., zeroth- and first-order in the magnitude of the wave vector, respectively. Black sticks at the top indicate the location of the underlying electronic transitions and black lines (solid and dashed) the experimental absorption spectra. ${ }^{83-87}$ Apart from uniform shifts necessary to align the lowestenergy band to the respective experimental spectrum, the theoretical spectra capture well both relative intensities and peak splittings.

The first valence band (Figure 1a) is dominated by the two excitations from the symmetric and antisymmetric combinations of the non-bonding $3 p$ orbital on each sulfur to the lowest unoccupied $\sigma_{\mathrm{SS}}^{*}$ orbital ( $b$ symmetry). This assignment is consistent with the analysis by Linderberg and Michl on $\mathrm{H}_{2} \mathrm{~S}_{2} \cdot{ }^{88}$ Since the valence orbitals are found to have well-defined 

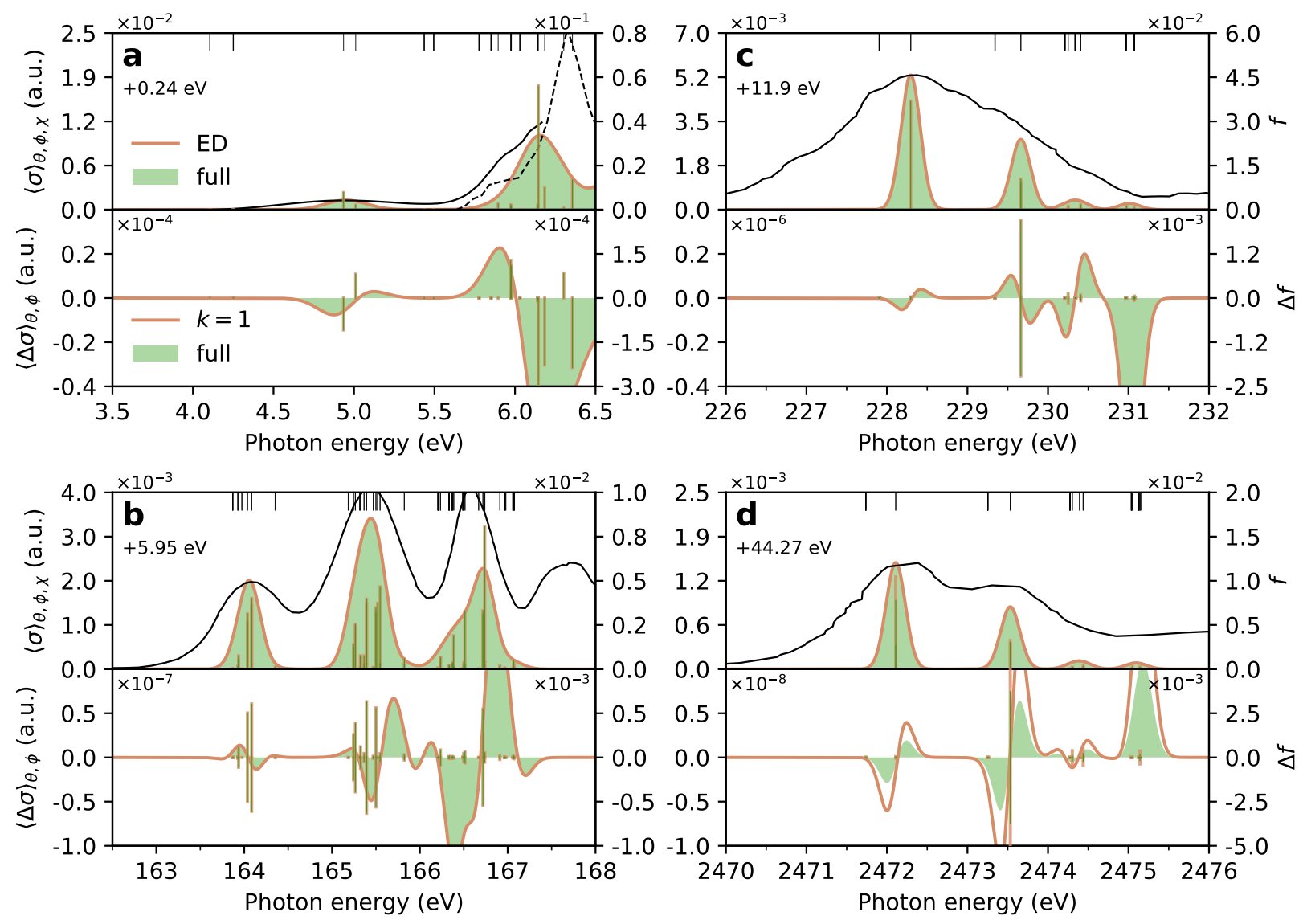

Figure 1: Isotropic linear (top) and differential (bottom) absorption spectra of $\left(\mathrm{CH}_{3} \mathrm{~S}\right)_{2}$ : (a) valence, (b) $L_{2,3}$-edge, (c) $L_{1}$-edge and (d) $K$-edge spectra using the full interaction operator in Eq. (3) (green shadings) or the lowest non-vanishing generalized velocity representation (orange lines). Left axes correspond to (differential) absorption cross-sections, whereas (differential) oscillator strengths (sticks) are shown on the right axes. Black sticks indicate the location of all computed transitions whereas black lines are experimental spectra. ${ }^{83-87}$ The stick spectra were convolved with a Gaussian lineshape with FWHM of $0.4 \mathrm{eV}$. The theoretical absorption spectra have been uniformly shifted to align with the experimental counterparts (shift values indicated in the top panels). The same shifts were applied to the ECD spectra.

spin, we adopt a non-relativistic state notation for the valence states, i.e., $1^{1} B$ and $2^{1} A$, respectively. They are separated by $\sim 0.15 \mathrm{eV}$. The second valence band originates from transitions into the $\sigma_{\mathrm{CS}}^{*}$ orbital $\left(3^{1} A\right.$ and $\left.2^{1} B\right)$.

Turning to the X-ray region, the first two bands at the sulfur $L_{2,3}$-edge (Figure 1b) are dominated by transitions from the symmetric and antisymmetric combinations of the $\mathrm{S}$ 
$2 p_{3 / 2}$ and $2 p_{1 / 2}$ core orbitals to the $\sigma_{\mathrm{SS}}^{*}$ orbital. We note that the $A / B$ pair of associated transitions are essentially degenerate (less than $0.1 \mathrm{meV}$ splitting) because of the limited overlap between the core orbitals on each of the sulfur atoms. The calculated spin-orbit splitting of $\sim 1.35 \mathrm{eV}$ between the $L_{3^{-}}$and $L_{2}$-branches is comparable to the experimental splitting reported for dihydrogen sulfide $\left(\sim 1.2 \mathrm{eV}^{89,90}\right)$. The $L_{3} / L_{2}$ branching ratio of $\sim 1.4: 1$ (obtained by summing the underlying oscillator strengths) deviates significantly from its statistical value, which is obtained only in the limit of $j-j$ coupling. ${ }^{91,92}$ The second peak in each branch is dominated by excitations to the $\sigma_{\mathrm{CS}}^{*}$ orbital and is separated from the first peak by $\sim 1.4-1.5 \mathrm{eV}$. Consequently, the second band in the spectrum contains contributions from both branches whereas the third band is associated with the second peak in the $L_{2}$ branch. These assignments agree with previous studies. ${ }^{85,93}$ The energy range considered as well as the basis set used in our calculations does not cover the fourth band in the experimental spectrum which, according to previous work, ${ }^{85}$ originates from excitations to higher-lying orbitals of mixed $\sigma_{\mathrm{CS}}^{*} /$ Rydberg character. Not surprisingly, the $L_{1^{-}}$and $K$-edge spectra bear strong resemblance (Figure 1c-d): they display two pre-edge features, separated by $\sim 1.5 \mathrm{eV}$, which originate from pairs of near-degenerate excitations from the bonding and antibonding combinations of sulfur $s$-orbitals into the $\sigma_{\mathrm{SS}}^{*}$ and $\sigma_{\mathrm{CS}}^{*}$ orbitals, respectively. ${ }^{86,94,95}$

A non-vanishing ECD response in these minimal disulfides results from axial chirality caused by trapping the disulfide bridge in a non-planar (i.e., $C_{2}$ ) conformation. As described above, the two lowest-energy transitions in each spectral domain are dominated by an excitation from the bonding or antibonding combinations of the relevant atomic orbitals on the sulfurs into the $\sigma_{\mathrm{SS}}^{*}$ orbital. This pairing of transitions manifests as bisignate features in the low-energy region of the ECD spectra. On the basis of the simple Bergson model for the low-energy transitions in the disulfide chromophore, ${ }^{96,97}$ Linderberg and Michl ${ }^{88}$ formulated a quadrant rule for the optical activity of the two low-energy valence transitions (dominated by excitations from the symmetric and antisymmetric combinations of non-bonding $3 p$ orbitals on sulfurs to the $\sigma_{\mathrm{SS}}^{*}$ orbital) in organic disulfides. This rule relates the sign of 
the long-wavelength Cotton effect across the four dihedral quadrants and is a specific case of the $C_{2}$-rule for general chromophores of effective $C_{2}$ symmetry. ${ }^{98}$ Woody extended the theoretical analysis to also include the absolute sign of the lowest ECD band within each quadrant, ${ }^{99}$ providing predictions in agreement with experimental results across different dihedral angles. ${ }^{100-103}$

For the $M$-helical form considered here, we find a negative-first Cotton effect, consistent with the quadrant rule. ${ }^{99}$ The intensity asymmetry of the lowest-energy valence couplet is attributed to different intrafragment (i.e., $\mathrm{CH}_{3} \mathrm{~S}-$ ) contributions to the ECD signal of each transition. At higher energies, the electronic coupling between the (core) orbitals decreases, reducing both their energetic splitting and the intrafragment ECD contributions which become increasingly atomic-like. As a consequence, the paired core transitions become neardegenerate (energy splitting of a few meV or less) with rotational strengths of almost equal magnitudes but opposite signs (see Table 1). Hence, after additionally accounting for sources of broadening, including finite core-hole lifetimes $(\sim 0.1$ and $\sim 0.5 \mathrm{eV}$ at the sulfur $L$ - and $K$-edges, ${ }^{104,105}$ respectively), we may realistically only be able to resolve the differential contributions with the absolute sign given by the most intense of the transitions. Accordingly, the effective signals are therefore reduced by orders of magnitude in the X-ray region (see accumulated values in Table 1).

The linear absorption profiles with the full interaction and the electric-dipole approximation essentially coincide across the four spectral regions. However, as shown by the underlying oscillator strengths in Table 1, non-dipolar effects at the $K$-edge lead to intensity redistribution among the underlying near-degenerate transitions (i.e., unrelated to the arbitrary mixing allowed for degenerate states). Nonetheless, the overall spectral profiles within and beyond the electric-dipole approximation are essentially identical because of the nearly overlapping transitions. This is consistent with our previous findings for $\mathrm{Cl} K$-edge absorption in $\mathrm{TiCl}_{4} \cdot{ }^{28}$ In contrast, the beyond-first-order effects become evident in the differential $K$-edge absorption profile because of the signed nature of the underlying quantities. 
This leads to a factor-of-two overestimation of the ECD within the conventional first-order treatment. As discussed below, introducing third-order contributions largely corrects this discrepancy.

To better understand the nature of the chiral response across spectral regions, we computed the underlying anisotropic differential oscillator strength distributions. Figure 2 shows the full ECD distributions (points), compared with the first-order truncated counterparts (surfaces), for transitions in $\mathrm{H}_{2} \mathrm{~S}_{2}$. The solid angle represents the propagation direction, the distance from the origin $(\mathrm{COM})$ indicates the magnitude of the associated signal and the color its sign. Note that different scaling factors have been applied across the transitions (see upper right corner of each subfigure). The $C_{2}$-rotation axis coincides with the $z$-axis, whereas the disulfide bond is along the $x$-axis. The shapes of the anisotropic distributions can be understood by decomposing the first-order signals into isotropic and $d$-orbital contributions (Eq. (26)). The resulting orbital weights of the excitations plotted in Figure 2 are reported in Table 2 .

From symmetry considerations detailed in the SI, we find that contributions from $d_{x z}$ and $d_{y z}$ vanish for excitations of both $A$ and $B$ symmetry. In $A$ symmetry, $R_{z z}^{[1]}$ is also zero by symmetry, such that the $s$ and $d_{z^{2}}$ contributions come in a fixed ratio, giving a toroid in the $x y$-plane. For valence and $L_{3}$-edge excitations of $A$ symmetry this shape is modulated by the $d_{x^{2}-y^{2}}$ contribution, giving the shape of a biconcave disc elongated along the $y$ - and $x$ axis, respectively, depending on its relative sign. For the $L_{1}$ - and $K$-edge excitations the $d_{x y}$ contribution completely dominates. This is also the case for the corresponding excitations in $B$ symmetry. It may be noted that in $A$ symmetry the E1-M1 contribution to the $d_{x y}$ term is zero by symmetry, whereas in $B$ symmetry the E1-E2 and E1-M1 contributions are of similar magnitude and the same sign. Continuing to the $L_{3}$ excitation of $B$ symmetry, the angular plot resembles that of its counterpart in $A$ symmetry, albeit with opposite overall sign and rotated $\pi / 2$ about the molecular axes. The latter can be understood from the relative weights of $d_{z^{2}}$ and $d_{x^{2}-y^{2}}$ contributions, as seen in Table 2. Finally, the angular 
plot of the valence excitation in $B$ symmetry is a biconcave disc elongated along the $z$-axis,

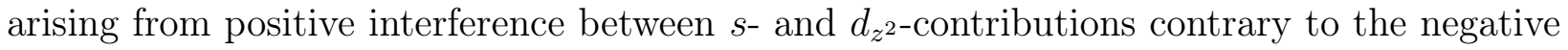
interference observed for its counterpart of $A$ symmetry.

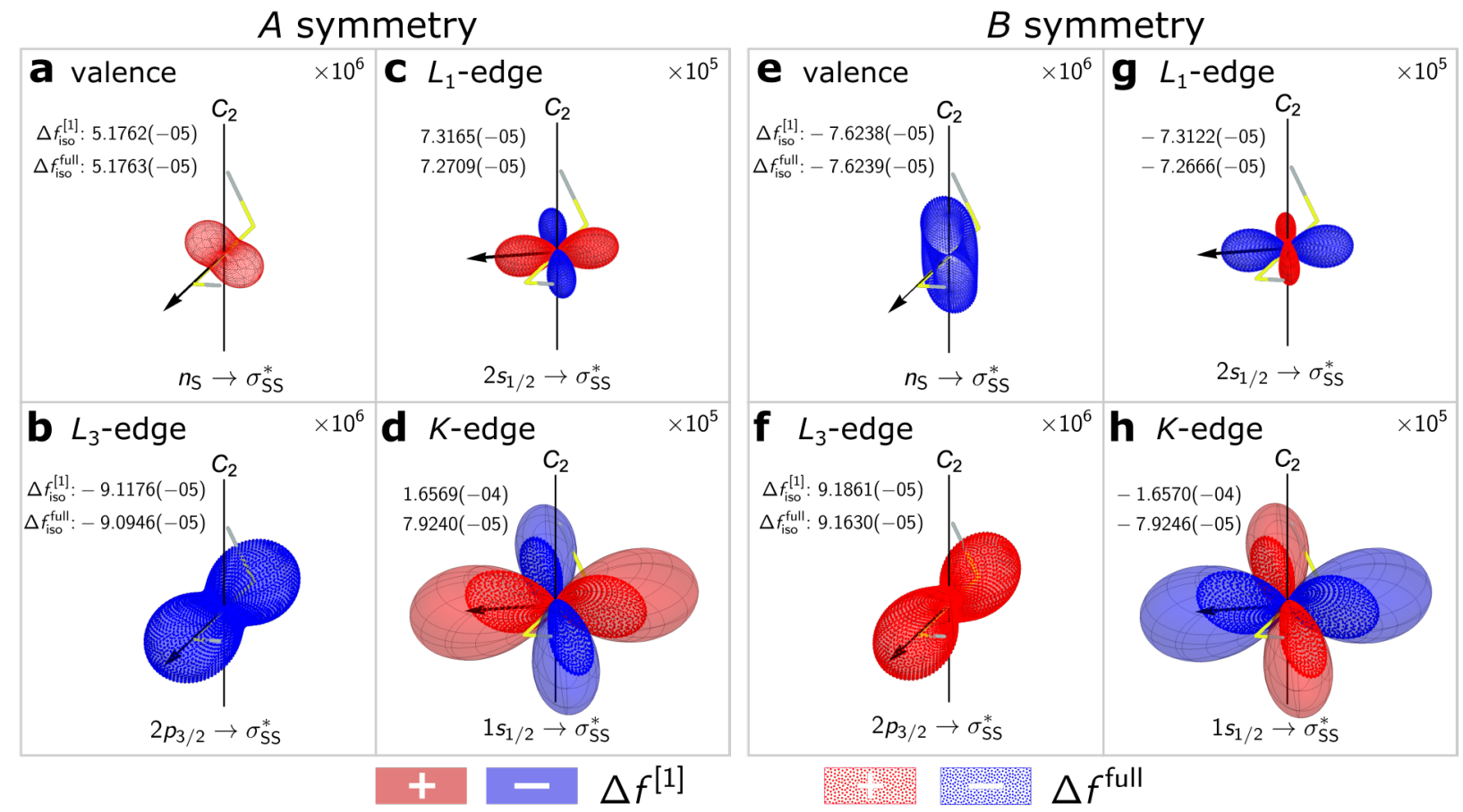

Figure 2: Comparison of full and truncated differential oscillator strength $\Delta f(\theta, \phi)$ across the spectral regions (valence, $L_{3^{-}}, L_{1^{-}}$and $K$-edges). Transitions of (a-d) $A$ and (e-h) $B$ symmetry in $\mathrm{H}_{2} \mathrm{~S}_{2}$. The black arrow points along the direction of the wave vector for the anisotropic ECD intensity given in Table 1. The truncated ECD is represented by the smooth surface, whereas the full ECD is shown as individual points generated with a 5810point Lebedev grid $\left(L_{\max }=131\right)$. Blue: negative; red: positive ECD signal. Note that different scaling factors (upper right corner) have been applied. The corresponding isotropic differential oscillator strengths are indicated in each subfigure.

Next, we compare these conventional first-order truncated ECD distributions with their full counterparts. For the valence, $L_{3}$ - and $L_{1}$-edge transitions, the anisotropic distributions virtually coincide, thereby confirming the validity of the first-order truncated description also for the anisotropic signal in these energy regimes. On the other hand, the full and truncated ECD distribution for the $K$-edge transitions are seen to have the same overall shape, but markedly different size, such that the factor-of-two overestimation at first order of the isotropic response (Table 1/S1) arises largely from an overall scaling. Closer inspection 


\section{A symmetry}
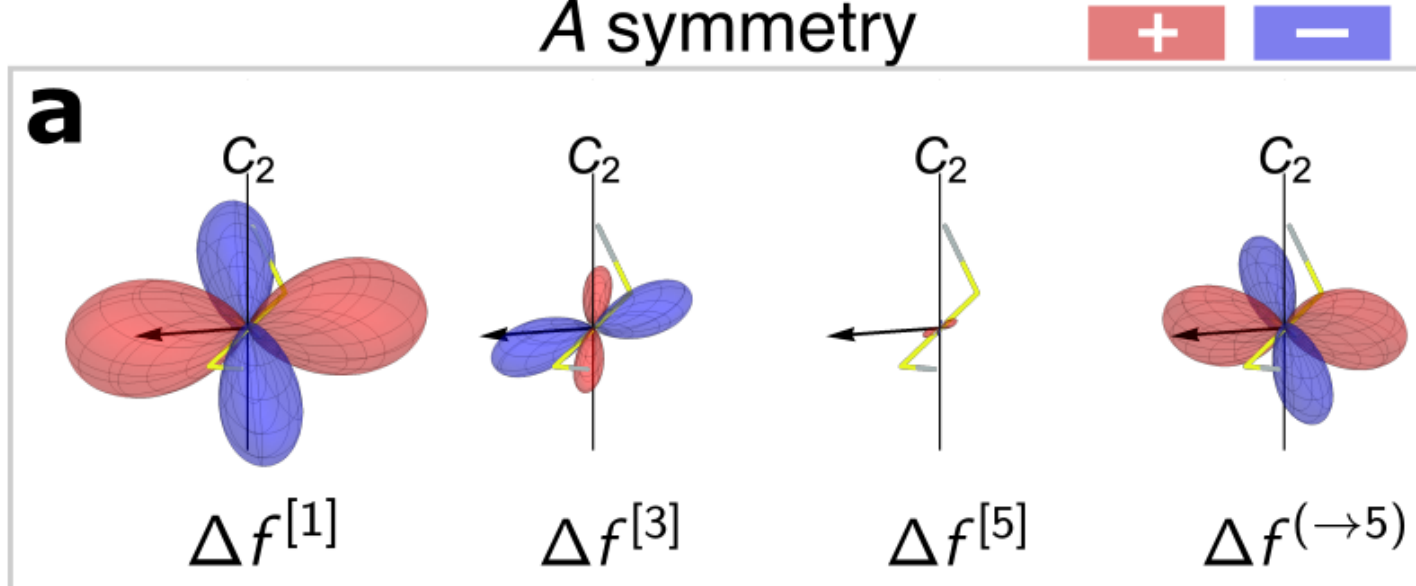

$\Delta f^{[3]}$

$\Delta f^{[5]}$

$\Delta f^{(\rightarrow 5)}$

\section{$B$ symmetry}

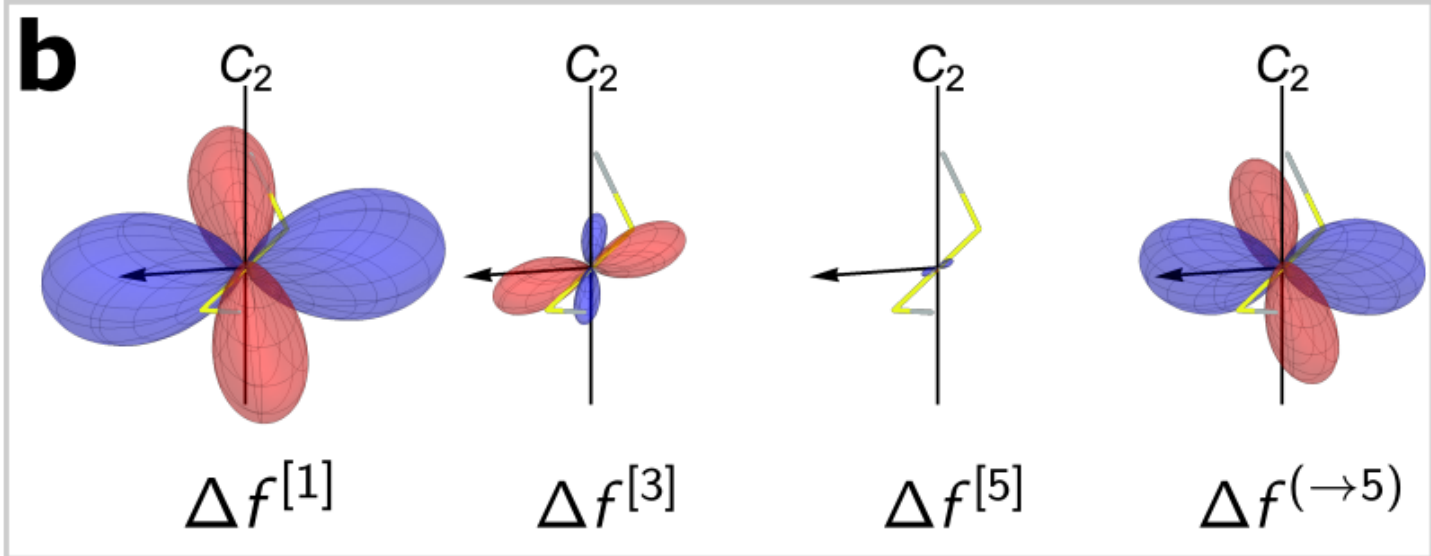

Figure 3: Convergence of the truncated anisotropic ECD distributions, i.e., $\Delta f^{[2 n+1]}(\theta, \phi)$, for the $K$-edge transitions in $\mathrm{H}_{2} \mathrm{~S}_{2}$. The black arrows point along the direction of the wave vector for the anisotropic ECD intensity given in Table S1.

of the angular distribution of the full ECD distribution reveals that the lobes are not strictly perpendicular as in a $d_{x y}$-orbital, hence indicating the contributions from solid harmonics of higher even angular momentum. To investigate this further, we have gathered the orderby-order contributions together with the full anisotropic ECD distribution in Figure 3. We indeed see that although $\Delta f^{[3]}(\ell=0,2,4)$ resembles $\Delta f^{[1]}$, the inclusion of higher-order solid harmonics in the former leads to non-orthogonal lobes. Furthermore, the two distributions differ by an overall sign, such that the inclusion of $\Delta f^{[3]}$ decreases the ECD signal. A possible issue is the rate of convergence of the truncated interaction towards the full one. 
Our implementation of truncated interaction for linear absorption allows us to go to arbitrary order, a unique functionality of the DIRAC code. In our previous work, ${ }^{28}$ we were able to demonstrate that the truncated treatment converges to the full interaction upon inclusion of higher-order terms, but for highly energetic transitions (photon energies beyond $\sim 3728$ $\mathrm{eV}$ ) the convergence behavior was too slow for practical applications. In the present case, we assessed the convergence of the isotropic differential oscillator strength expansion at the sulfur $K$-edge and found that the relative error, reported in Table 3, is below the threshold of $1 \%$ at 7 th order. Indeed, one sees in Figure 3 that the $\Delta f^{[5]}$ distribution $(\ell=0,2,4,6)$ is minute. We also assessed the convergence of the expanded isotropic linear oscillator strength, which reaches the same threshold at an equivalent rate.

\section{Conclusion}

We have reported the first implementation and application of the anisotropic and isotropic ECD signal using the full semi-classical light-matter interaction operator within a fourcomponent relativistic framework. This simultaneous account of beyond-first-order lightmatter interactions and relativistic effects provides two additional sources of ECD which become increasingly important at high photon energies. The linear form of the light-matter interaction operator in the relativistic domain further enabled straightforward extension to a multipole-based scheme in the velocity representation that allows for the traditional (albeit, in general, ambiguous) decomposition into electric and magnetic contributions while retaining order-by-order gauge-origin independence.

The presented approach was used to investigate the ECD response of two prototypical disulfides, $\mathrm{H}_{2} \mathrm{~S}_{2}$ and $\left(\mathrm{CH}_{3} \mathrm{~S}\right)_{2}$, across the electromagnetic spectrum, from valence to core transitions. To quantify the implications of higher-order effects, we compared the results of the full interaction to those obtained within the traditional lowest-order non-vanishing (i.e., first-order) truncated generalized velocity representation. Going beyond the electric-dipole 
approximation at the sulfur $K$-edge leads to non-negligible intensity redistribution among near-degenerate transitions but with no visible implications on the linear absorption profile. On the other hand, the differential absorption profile is affected by such redistribution, because of its signed nature. This leads to an overall factor-of-two overestimation. By examining the shapes of the underlying anisotropic ECD distributions, we find this discrepancy to largely originate from an overall scaling that is corrected upon introducing third-order contributions.

From a practical point of view, the full interaction operator provides a compact and inherently gauge-origin invariant treatment of light-matter interactions which is both implementationand computation-wise competitive with traditional multipole-based schemes for oriented and isotropic linear spectroscopies. For this reason, we believe that the use of the full light-matter interaction will become the new standard for theoretical X-ray spectroscopies. The question of how to efficiently handle the rotational averaging for nonlinear light-matter interactions will be explored in future work.

\section{Acknowledgement}

N.H.L. acknowledges financial support from the Carlsberg Foundation (Grant No. CF160290). M.v.H acknowledges funding from the French Ministry of Higher Education and Research. Computing time from CALMIP (Calcul en Midi-Pyrenées) and SNIC (Swedish National Infrastructure for Computing) at National Supercomputer Centre (NSC) are gratefully acknowledged.

\section{Supporting Information Available}

Linear and differential absorption spectra and corresponding tabulated oscillator strengths for $\mathrm{H}_{2} \mathrm{~S}_{2}$. Analysis of structure and origin dependence of the rotational strength tensor. 


\section{References}

(1) Dorta-Urra, A.; Bargueño, P. Homochirality: A perspective from fundamental physics. Symmetry 2019, 11, 661, DOI: doi:10.3390/sym11050661.

(2) Barron, L. D. Molecular light scattering and optical activity; Cambridge University Press, 2004.

(3) Schipper, P. E.; Rodger, A. Symmetry rules for the determination of the intercalation geometry of host/systems using circular dichroism: a symmetry-adapted coupledoscillator model. Journal of the American Chemical Society 1983, 105, 4541-4550, DOI: doi:10.1021/ja00352a007.

(4) Holmgaard List, N.; Knoops, J.; Rubio-Magnieto, J.; Idé, J.; Beljonne, D.; Norman, P.; Surin, M.; Linares, M. Origin of DNA-induced circular dichroism in a minor-groove binder. Journal of the American Chemical Society 2017, 139, 14947-14953, DOI: doi:10.1021/jacs.7b05994.

(5) Berova, N.; Nakanishi, K.; Woody, R. W. Circular dichroism: principles and applications; John Wiley \& Sons, 2000.

(6) Lindorfer, D.; Renger, T. Theory of Anisotropic Circular Dichroism of Excitonically Coupled Systems: Application to the Baseplate of Green Sulfur Bacteria. The Journal of Physical Chemistry B 2018, 122, 2747-2756, DOI: doi:10.1021/acs.jpcb.7b12832.

(7) Akhtar, P.; Lindorfer, D.; Lingvay, M.; Pawlak, K.; Zsiros, O.; Siligardi, G.; Jávorfi, T.; Dorogi, M.; Ughy, B.; Garab, G.; Renger, T.; Lambrev, P. H. Anisotropic Circular Dichroism of Light-Harvesting Complex II in Oriented Lipid Bilayers: Theory Meets Experiment. The Journal of Physical Chemistry B 2019, 123, 1090-1098, DOI: doi:10.1021/acs.jpcb.8b12474. 
(8) Arago, M. Mémoire sur une modification remarquable qu'éprouvent les rayons lumineux dans leur passage à travers certains corps diaphanes et sur quelques autres nouveaux phénomènes d'optique. Mémoires de l'Institut de France 1811, 1, 93-134.

(9) Biot, J.-B. Mémoire sur un nouveau genre d'oscillations que les molecules de la lumière éprouvent, en traversant certains cristaux. Mémoires de l'Institut de France 1812, 1, 1-372.

(10) Alagna, L.; Prosperi, T.; Turchini, S.; Goulon, J.; Rogalev, A.; Goulon-Ginet, C.; Natoli, C. R.; Peacock, R. D.; Stewart, B. X-ray natural circular dichroism. Physical Review Letters 1998, 80, 4799, DOI: doi:10.1103/PhysRevLett.80.4799.

(11) Goulon, J.; Goulon-Ginet, C.; Rogalev, A.; Gotte, V.; Malgrange, C.; Brouder, C.; Natoli, C. R. X-ray natural circular dichroism in a uniaxial gyrotropic single crystal of $\mathrm{LiIO}_{3}$. The Journal of Chemical Physics 1998, 108, 6394-6403, DOI: doi:10.1063/1.476046.

(12) Goulon, J.; Goulon-Ginet, C.; Rogalev, A.; Gotte, V.; Malgrange, C.; Brouder, C. Xray natural circular dichroism of gyrotropic crystals. Journal of synchrotron radiation 1999, 6, 673-675, DOI: doi:10.1117/12.370104.

(13) Stewart, B.; Peacock, R. D.; Alagna, L.; Prosperi, T.; Turchini, S.; Goulon, J.; Rogalev, A.; Goulon-Ginet, C. Circular Dichroism at the Edge: Large X-ray Natural $\mathrm{CD}$ in the $1 \mathrm{~s} \rightarrow 3 \mathrm{~d}$ Pre-Edge Feature of $2\left[\mathrm{Co}(\mathrm{en})_{3} \mathrm{Cl}_{3}\right] \cdot \mathrm{NaCl} \cdot 6 \mathrm{H}_{2} \mathrm{O}$. Journal of the American Chemical Society 1999, 121, 10233-10234, DOI: doi:10.1021/ja9920720.

(14) Tanaka, M.; Nakagawa, K.; Agui, A.; Fujii, K.; Yokoya, A. First observation of natural circular dichroism for biomolecules in soft X-ray region studied with a polarizing undulator. Physica Scripta 2005, 2005, 873, DOI: doi:10.1238/Physica.Topical.115a00873.

(15) Nakagawa, K.; Matsui, T.; Izumi, Y.; Agui, A.; Tanaka, M.; Muro, T. Radiation- 
induced chemical evolution of biomolecules. Radiation Physics and Chemistry 2009, 78, 1198 - 1201, DOI: doi:10.1016/j.radphyschem.2009.07.010.

(16) Izumi, Y.; Imazu, A.; Mimoto, A.; Tanaka, M.; Nakagawa, K.; Tanaka, M.; Agui, A.; Muro, T. Measurement and comparison of absolute value of soft X-ray natural circular dichroism of serine and alanine. Journal of Physics: Conference Series 2009, 190, 012209, DOI: doi:10.1088/1742-6596/190/1/012209.

(17) Izumi, Y.; Tanabe, M.; Imazu, A.; Mimoto, A.; Tanaka, M.; Agui, A.; Muro, T.; Nakagawa, K. Characteristic oxygen K-edge circular dichroism spectra of amino acid films by improved measurement technique. The Journal of Chemical Physics 2013, 138, 074305, DOI: doi:10.1063/1.4790599.

(18) Goulon, J.; Sette, F.; Moise, C.; Fontaine, A.; Perey, D.; Rudolf, P.; Baudelet, F. Detection Limits for Natural Circular Dichroism of Chiral Complexes in the X-ray Range. Japanese Journal of Applied Physics 1993, 32, 284, DOI: doi:10.7567/jjaps.32s2.284.

(19) Rogalev, A.; Goulon, J.; Wilhelm, F.; Bosak, A. In Magnetism and Synchrotron Radiation; Beaurepaire, E., Bulou, H., Scheurer, F., Jean-Paul, K., Eds.; Springer Berlin Heidelberg: Berlin, Heidelberg, 2010; pp 169-190, DOI: doi:10.1007/978-3-642-04498$4 \_6$.

(20) Brouder, C. Angular dependence of X-ray absorption spectra. Journal of Physics: Condensed Matter 1990, 2, 701-738, DOI: doi:10.1088/0953-8984/2/3/018.

(21) Goulon, J. In Rayonnement synchrotron polarisé, électrons polarisés et magnétisme; Beaurepaire, E., Carrière, B., Kappler, J.-P., Eds.; IPCMS: Mittelwihr(Haut-Rhin), 1990; pp 333-386.

(22) Turchini, S.; Zema, N.; Zennaro, S.; Alagna, L.; Stewart, B.; Peacock, R. D.; Prosperi, T. Core Electron Transitions as a Probe for Molecular Chirality: Natural Circular 
Dichroism at the Carbon K-edge of Methyloxirane. Journal of the American Chemical Society 2004, 126, 4532-4533, DOI: doi:10.1021/ja039348v.

(23) Hansen, A. E.; Bouman, T. D. Advances in Chemical Physics; John Wiley \& Sons, Ltd, 2007; pp 545-644, DOI: doi:10.1002/9780470142639.ch5.

(24) London, F. Quantum theory of interatomic currents in aromatic compounds. J. Phys. Radium 1937, 8, 397-409, DOI: doi:10.1051/jphysrad:01937008010039700.

(25) Bak, K. L.; Hansen, A. E.; Ruud, K.; Helgaker, T.; Olsen, J.; Jørgensen, P. Ab initio calculation of electronic circular dichroism for trans-cyclooctene using London atomic orbitals. Theoretica chimica acta 1995, 90, 441-458, DOI: doi:10.1007/BF01113546.

(26) List, N. H.; Kauczor, J.; Saue, T.; Jensen, H. J. A.; Norman, P. Beyond the electricdipole approximation: A formulation and implementation of molecular response theory for the description of absorption of electromagnetic field radiation. The Journal of Chemical Physics 2015, 142, 244111, DOI: doi:10.1063/1.4922697.

(27) List, N. H.; Saue, T.; Norman, P. Rotationally averaged linear absorption spectra beyond the electric-dipole approximation. Molecular Physics 2017, 115, 63-74, DOI: doi:10.1080/00268976.2016.1187773.

(28) List, N. H.; Melin, T. R. L.; van Horn, M.; Saue, T. Beyond the electric-dipole approximation in simulations of x-ray absorption spectroscopy: Lessons from relativistic theory. The Journal of Chemical Physics 2020, 152, 184110, DOI: doi:10.1063/5.0003103.

(29) Villaume, S.; Norman, P. On circular dichroism and the separation between chromophore and chiral center: The near carbon K-edge X-ray absorption and circular dichroism spectra of noradrenaline and 1-DOPA. Chirality 2009, 21, E13-E19, DOI: doi:10.1002/chir.20763. 
(30) Zhang, Y.; Rouxel, J. R.; Autschbach, J.; Govind, N.; Mukamel, S. X-ray circular dichroism signals: a unique probe of local molecular chirality. Chemical science $\mathbf{2 0 1 7}$, 8, 5969-5978, DOI: doi:10.1039/C7SC01347G.

(31) Takahashi, O.; Kimoto, M.; Pettersson, L. G. Theoretical study of the X-ray natural circular dichroism of some crystalline amino acids. Chemical Physics 2015, 450, 109114, DOI: doi:10.1016/j.chemphys.2015.01.012.

(32) Kimberg, V.; Kosugi, N. Calculation of K-edge circular dichroism of amino acids: Comparison of random phase approximation with other methods. The Journal of Chemical Physics 2007, 126, 06B612, DOI: doi:10.1063/1.2743010.

(33) Carravetta, V.; Plachkevytch, O.; Vahtras, O.; Agren, H. Ordinary and rotatory intensities for X-ray absorption at the C-1s edge of organic chiral molecules: propylene oxide and trans-1,2-dimethylcyclopropane. Chemical Physics Letters 1997, 275, 70 78, DOI: doi:10.1016/S0009-2614(97)00687-8.

(34) Plashkevych, O.; Carravetta, V.; Vahtras, O.; Ågren, H. Theoretical study of Xray circular dichroism of amino acids. Chemical Physics 1998, 232, 49 - 62, DOI: doi:10.1016/S0301-0104(98)00055-X.

(35) Jiemchooroj, A.; Ekström, U.; Norman, P. Near-edge x-ray absorption and natural circular dichroism spectra of L-alanine: A theoretical study based on the complex polarization propagator approach. The Journal of Chemical Physics 2007, 127, 165104, DOI: doi:10.1063/1.2800024.

(36) Kaniauskas, J. Relativistic investigation of electronic transitions in many-electron atoms. Liet. Fiz. Rink 1974, XIV, 463-475, (in Russian; English translation available from the present authors).

(37) Grant, I. P. Relativistic quantum theory of atoms and molecules: theory and computation; Springer Science \& Business Media, 2007; Vol. 40; pp 673-713. 
(38) Urban, S.; Herbst, E.; Mittler, P.; Winnewisser, G.; Yamada, K. M.; Winnewisser, M. The high-resolution rotational and torsional spectra of HSSH. Journal of Molecular Spectroscopy 1989, 137, 327-353, DOI: doi:https://doi.org/10.1016/00222852(89)90177-X.

(39) Herbst, E.; Winnewisser, G. An experimentally derived torsional potential function for HSSH. Chemical Physics Letters 1989, 155, 572-575, DOI: doi:https://doi.org/10.1016/0009-2614(89)87475-5.

(40) Maciel, G. S.; Barreto, P. R. P.; Palazzetti, F.; Lombardi, A.; Aquilanti, V. A quantum chemical study of $\mathrm{H}_{2} \mathrm{~S}_{2}$ : Intramolecular torsional mode and intermolecular interactions with rare gases. The Journal of Chemical Physics 2008, 129, 164302, DOI: doi:10.1063/1.2994732.

(41) Aquilanti, V.; Ragni, M.; Bitencourt, A. C. P.; Maciel, G. S.; Prudente, F. V. Intramolecular Dynamics of RS-SR' Systems $\left(\mathrm{R}, \mathrm{R}\right.$ ' $\left.=\mathrm{H}, \mathrm{F}, \mathrm{Cl}, \mathrm{CH}_{3}, \mathrm{C}_{2} \mathrm{H}_{5}\right)$ : Torsional Potentials, Energy Levels, Partition Functions. The Journal of Physical Chemistry A 2009, 113, 3804-3813, DOI: doi:10.1021/jp8094215.

(42) Thornton, J. Disulphide bridges in globular proteins. Journal of Molecular Biology 1981, 151, 261-287, DOI: doi:10.1016/0022-2836(81)90515-5.

(43) Rauk, A. Chiroptical properties of disulfides. Ab initio studies of dihydrogen disulfide and dimethyl disulfide. Journal of the American Chemical Society 1984, 106, 65176524, DOI: doi:10.1021/ja00334a010.

(44) Ha, T.-K.; Cencek, W. Ab initio CI study of the optical rotatory strengths of HSSH. Chemical Physics Letters 1991, 182, 519-523, DOI: doi:https://doi.org/10.1016/00092614(91)90117-R.

(45) Pericou-Cayere, M.; Rerat, M.; Dargelos, A. Theoretical treatment of the electronic 
circular dichroism spectrum and the optical rotatory power of $\mathrm{H}_{2} \mathrm{~S}_{2}$. Chemical Physics 1998, 226, 297 - 306, DOI: doi:10.1016/S0301-0104(97)00285-1.

(46) Diedrich, C.; Grimme, S. Systematic Investigation of Modern Quantum Chemical Methods to Predict Electronic Circular Dichroism Spectra. The Journal of Physical Chemistry A 2003, 107, 2524-2539, DOI: doi:10.1021/jp0275802.

(47) Pecul, M.; Ruud, K. In Response Theory and Molecular Properties (A Tribute to Jan Linderberg and Poul Jørgensen); Jensen, H. J. Aa., Ed.; Advances in Quantum Chemistry; Academic Press, 2005; Vol. 50; pp 185-212, DOI: doi:https://doi.org/10.1016/S0065-3276(05)50009-1.

(48) Bednárová, L.; Bour̆, P.; Malon̆, P. Vibrational and electronic optical activity of the chiral disulphide group: Implications for disulphide bridge conformation. Chirality 2010, 22, 514-526, DOI: doi:10.1002/chir.20772.

(49) Scott, M.; Rehn, D. R.; Coriani, S.; Norman, P.; Dreuw, A. Electronic circular dichroism spectra using the algebraic diagrammatic construction schemes of the polarization propagator up to third order. The Journal of Chemical Physics 2021, 154, 064107, DOI: doi:10.1063/5.0038315.

(50) DIRAC, a relativistic ab initio electronic structure program, Release DIRAC21 (2021), written by R. Bast, A. S. P. Gomes, T. Saue, L. Visscher, and H. J. Aa. Jensen, with contributions from I. A. Aucar, V. Bakken, K. G. Dyall, S. Dubillard, U. Ekström, E. Eliav, T. Enevoldsen, E. Faßhauer, T. Fleig, O. Fossgaard, L. Halbert, E. D. Hedegård, T. Helgaker, B. Helmich-Paris, J. Henriksson, M. Iliaš, Ch. R. Jacob, S. Knecht, S. Komorovský, O. Kullie, J. K. Lærdahl, C. V. Larsen, Y. S. Lee, N. H. List, H. S. Nataraj, M. K. Nayak, P. Norman, G. Olejniczak, J. Olsen, J. M. H. Olsen, A. Papadopoulos, Y. C. Park, J. K. Pedersen, M. Pernpointner, J. V. Pototschnig, R. di Remigio, M. Repisky, K. Ruud, P. Sałek, B. Schimmelpfennig, B. Senjean, 
A. Shee, J. Sikkema, A. Sunaga, A. J. Thorvaldsen, J. Thyssen, J. van Stralen, M. L. Vidal, S. Villaume, O. Visser, T. Winther, and S. Yamamoto (available at http: //dx.doi.org/10.5281/zenodo.4836496, see also http://www. diracprogram.org).

(51) Saue, T.; Bast, R.; Gomes, A. S. P.; Jensen, H. J. Aa.; Visscher, L.; Aucar, I. A.; Di Remigio, R.; Dyall, K. G.; Eliav, E.; Fasshauer, E.; Fleig, T.; Halbert, L.; Hedegård, E. D.; Helmich-Paris, B.; Iliaš, M.; Jacob, C. R.; Knecht, S.; Laerdahl, J. K.; Vidal, M. L.; Nayak, M. K.; Olejniczak, M.; Olsen, J. M. H.; Pernpointner, M.; Senjean, B.; Shee, A.; Sunaga, A.; van Stralen, J. N. P. The DIRAC code for relativistic molecular calculations. The Journal of Chemical Physics 2020, 152, 204104, DOI: doi:10.1063/5.0004844.

(52) Braslavsky, S. E. Glossary of terms used in photochemistry, 3rd edition (IUPAC Recommendations 2006). Pure and Applied Chemistry 2007, 79, 293 - 465, DOI: doi:10.1351/pac200779030293.

(53) Hansen, A.; Avery, J. A fully retarded expression for the rotatory strength. Chemical Physics Letters 1972, 13, 396 - 398, DOI: doi:10.1016/0009-2614(72)80109-X.

(54) Bloch., F. In W. Heisenberg und die Physik unserer Zeit; Bopp, F., Ed.; Vieweg \& Sohn: Braunschweig, 1961.

(55) Kobe, D. H. Gauge transformations and the electric dipole approximation. Am. J. Phys. 1982, 50, 128, DOI: doi:10.1119/1.13029.

(56) Stewart, A. M. General proof of gauge independence of the sum of the dia- and paramagnetic magnetic moments. Journal of Physics A: Mathematical and General 1999, 32, 6091, DOI: doi:10.1088/0305-4470/32/33/307.

(57) Saue, T. In Relativistic Electronic Structure Theory. Part 1. Fundamentals; Schwerdtfeger, P., Ed.; Elsevier: Amsterdam, 2002; p 332. 
(58) Norman, P.; Ruud, K.; Saue, T. Principles and Practices of Molecular Properties: Theory, Modeling and Simulations; Wiley: Hoboken, NJ, 2018; DOI: doi:10.1002/9781118794821.

(59) Pedersen, T. B.; Hansen, A. E. Ab initio calculation and display of the rotary strength tensor in the random phase approximation. Method and model studies. Chemical Physics Letters 1995, 246, 1-8, DOI: doi:10.1016/0009-2614(95)01036-9.

(60) Kuball, H.-G.; Sieber, G.; Neubrech, S.; Schultheis, B.; Schönhofer, A. Circular dichroism of oriented molecules. Magnetic dipole and electric quadrupole contribution to the ACD of chirally substituted diaminoanthraquinones. Chemical Physics 1993, 169, 335 - 350, DOI: doi:10.1016/0301-0104(93)85104-G.

(61) Saue, T.; Jensen, H. J. A. Quaternion symmetry in relativistic molecular calculations: The Dirac-Hartree-Fock method. The Journal of Chemical Physics 1999, 111, 62116222, DOI: doi:10.1063/1.479958.

(62) Stephens, P. J.; Devlin, F. J.; Chabalowski, C. F.; Frisch, M. J. Ab Initio Calculation of Vibrational Absorption and Circular Dichroism Spectra Using Density Functional Force Fields. J. Phys. Chem. 1994, 98, 11623-11627, DOI: doi:10.1021/j100096a001.

(63) Becke, A. D. Density-Functional Thermochemistry. III. The Role of Exact Exchange. J. Chem. Phys. 1993, 98, 5648-5652, DOI: doi:10.1063/1.464913.

(64) Vosko, S. H.; Wilk, L.; Nusair, M. Accurate Spin-Dependent Electron Liquid Correlation Energies for Local Spin Density Calculations: A Critical Analysis. Can. J. Phys. 1980, 58, 1200-1211, DOI: doi:10.1139/p80-159.

(65) Lee, C.; Yang, W.; Parr, R. G. Development of the Colle-Salvetti Correlation-Energy Formula Into a Functional of the Electron Density. Phys. Rev. B 1988, 37, 785-789, DOI: doi:10.1103/PhysRevB.37.785. 
(66) Dunning, T. H. Gaussian Basis Sets for Use in Correlated Molecular Calculations. I. The Atoms Boron through Neon and Hydrogen. J. Chem. Phys. 1989, 90, 1007-1023, DOI: doi:10.1063/1.456153.

(67) Woon, D. E.; Dunning, T. H. Gaussian basis sets for use in correlated molecular calculations. III. The atoms aluminum through argon. The Journal of Chemical Physics 1993, 98, 1358-1371, DOI: doi:10.1063/1.464303.

(68) Frisch, M. J.; Trucks, G. W.; Schlegel, H. B.; Scuseria, G. E.; Robb, M. A.; Cheeseman, J. R.; Scalmani, G.; Barone, V.; Petersson, G. A.; Nakatsuji, H.; Li, X.; Caricato, M.; Marenich, A. V.; Bloino, J.; Janesko, B. G.; Gomperts, R.; Mennucci, B.; Hratchian, H. P.; Ortiz, J. V.; Izmaylov, A. F.; Sonnenberg, J. L.; WilliamsYoung, D.; Ding, F.; Lipparini, F.; Egidi, F.; Goings, J.; Peng, B.; Petrone, A.; Henderson, T.; Ranasinghe, D.; Zakrzewski, V. G.; Gao, J.; Rega, N.; Zheng, G.; Liang, W.; Hada, M.; Ehara, M.; Toyota, K.; Fukuda, R.; Hasegawa, J.; Ishida, M.; Nakajima, T.; Honda, Y.; Kitao, O.; Nakai, H.; Vreven, T.; Throssell, K.; Montgomery, J. A., Jr.; Peralta, J. E.; Ogliaro, F.; Bearpark, M. J.; Heyd, J. J.; Brothers, E. N.; Kudin, K. N.; Staroverov, V. N.; Keith, T. A.; Kobayashi, R.; Normand, J.; Raghavachari, K.; Rendell, A. P.; Burant, J. C.; Iyengar, S. S.; Tomasi, J.; Cossi, M.; Millam, J. M.; Klene, M.; Adamo, C.; Cammi, R.; Ochterski, J. W.; Martin, R. L.; Morokuma, K.; Farkas, O.; Foresman, J. B.; Fox, D. J. Gaussian16 Revision C.01. 2016; Gaussian Inc. Wallingford CT.

(69) Craig, D. B.; Dombkowski, A. A. Disulfide by Design 2.0: a web-based tool for disulfide engineering in proteins. BMC bioinformatics 2013, 14, 346, DOI: doi:10.1186/14712105-14-346.

(70) Pescitelli, G.; Di Bari, L.; Berova, N. Conformational aspects in the studies of organic compounds by electronic circular dichroism. Chem. Soc. Rev. 2011, 40, 4603-4625, DOI: doi:10.1039/C1CS15036G. 
(71) Stener, M.; Fronzoni, G.; de Simone, M. Time dependent density functional theory of core electrons excitations. Chem. Phys. Lett. 2003, 373, 115 - 123, DOI: doi:10.1016/S0009-2614(03)00543-8.

(72) South, C.; Shee, A.; Mukherjee, D.; Wilson, A. K.; Saue, T. 4-Component relativistic calculations of $\mathrm{L}_{3}$ ionization and excitations for the isoelectronic species $\mathrm{UO}_{2}^{2+} \mathrm{OUN}^{+}$and $\mathrm{UN}_{2}$. Phys. Chem. Chem. Phys. 2016, 18, 21010-21023, DOI: doi:10.1039/C6CP00262E.

(73) Kadek, M.; Konecny, L.; Gao, B.; Repisky, M.; Ruud, K. X-ray absorption resonances near $\mathrm{L}_{2,3}$-edges from real-time propagation of the Dirac-Kohn-Sham density matrix. Phys. Chem. Chem. Phys. 2015, 17, 22566-22570, DOI: doi:10.1039/C5CP03712C.

(74) Fransson, T.; Burdakova, D.; Norman, P. K- and L-edge X-ray absorption spectrum calculations of closed-shell carbon, silicon, germanium, and sulfur compounds using damped four-component density functional response theory. Phys. Chem. Chem. Phys. 2016, 18, 13591-13603, DOI: doi:10.1039/C6CP00561F.

(75) Visscher, L.; Dyall, K. G. Dirac-Fock atomic electronic structure calculations using different nuclear charge distributions. At. Data Nucl. Data Tables 1997, 67, 207, DOI: doi:10.1006/adnd.1997.0751.

(76) Perdew, J. P.; Burke, K.; Ernzerhof, M. Generalized gradient approximation made simple. Physical Review Letters 1996, 77, 3865, DOI: doi:10.1103/PhysRevLett.77.3865.

(77) Adamo, C.; Barone, V. Toward reliable density functional methods without adjustable parameters: The PBE0 model. The Journal of chemical physics 1999, 110, 6158-6170, DOI: doi:10.1063/1.478522.

(78) Ambroise, M. A.; Jensen, F. Probing Basis Set Requirements for Calculating Core Ionization and Core Excitation Spectroscopy by the $\Delta$ Self-Consistent-Field Ap- 
proach. Journal of Chemical Theory and Computation 2019, 15, 325-337, DOI: doi:10.1021/acs.jctc.8b01071.

(79) Jensen, F. Polarization consistent basis sets: Principles. The Journal of Chemical Physics 2001, 115, 9113-9125, DOI: doi:10.1063/1.1413524.

(80) Jensen, F. Polarization consistent basis sets. II. Estimating the Kohn-Sham basis set limit. The Journal of Chemical Physics 2002, 116, 7372-7379, DOI: doi:10.1063/1.1465405.

(81) Jensen, F. Polarization consistent basis sets. III. The importance of diffuse functions. The Journal of Chemical Physics 2002, 117, 9234-9240, DOI: doi:10.1063/1.1515484.

(82) Visscher, L. Approximate molecular relativistic Dirac-Coulomb calculations using a simple Coulombic correction. Theoretical Chemistry Accounts 1997, 98, 68-70, DOI: doi:10.1007/s002140050280.

(83) Tokue, I.; Hiraya, A.; Shobatake, K. Photoexcitation of dimethyl sulfide and dimethyl disulfide in the vacuum ultraviolet region: Rydberg states and photofragment emissions. Chemical Physics 1989, 130, 401-408, DOI: doi:10.1016/0301-0104(89)87069-7.

(84) Hearn, C. H.; Turcu, E.; Joens, J. A. The near U.V. absorption spectra of dimethyl sulfide, diethyl sulfide and dimethyl disulfide at $\mathrm{T}=300 \mathrm{~K}$. Atmospheric Environment. Part A. General Topics 1990, 24, 1939-1944, DOI: doi:https://doi.org/10.1016/09601686(90)90527-T.

(85) Schnorr, K.; Bhattacherjee, A.; Oosterbaan, K. J.; Delcey, M. G.; Yang, Z.; Xue, T.; Attar, A. R.; Chatterley, A. S.; Head-Gordon, M.; Leone, S. R.; Gessner, O. Tracing the 267 nm-Induced Radical Formation in Dimethyl Disulfide Using Time-Resolved X-ray Absorption Spectroscopy. The Journal of Physical Chemistry Letters 2019, 10, 1382-1387, DOI: doi:10.1021/acs.jpclett.9b00159. 
(86) Ochmann, M.; Hussain, A.; von Ahnen, I.; Cordones, A. A.; Hong, K.; Lee, J. H.; Ma, R.; Adamczyk, K.; Kim, T. K.; Schoenlein, R. W.; Vendrell, O.; Huse, N. UVPhotochemistry of the Disulfide Bond: Evolution of Early Photoproducts from Picosecond X-ray Absorption Spectroscopy at the Sulfur K-Edge. Journal of the American Chemical Society 2018, 140, 6554-6561, DOI: doi:10.1021/jacs.7b13455, PMID: 29771112.

(87) Hitchcock, A.; Bodeur, S.; Tronc, M. Sulfur and Chlorine K-shell spectra of gases. Physica B: Condensed Matter 1989, 158, 257-258, DOI: doi:https://doi.org/10.1016/0921-4526(89)90279-2.

(88) Linderberg, J.; Michl, J. Inherent optical activity of organic disulfides. J. Am. Chem. Soc. 1970, 92, 2619-2625, DOI: doi:10.1021/ja00712a005.

(89) Svensson, S.; Naves de Brito, A.; Keane, M. P.; Correia, N.; Karlsson, L. Observation of an energy shift in the $\mathrm{S} 2 p_{3 / 2}-\mathrm{S} 2 p_{1 / 2}$ spin-orbit splitting between x-ray photoelectron and Auger-electron spectra for the $\mathrm{H}_{2} \mathrm{~S}$ molecule. Phys. Rev. A 1991, 43, 6441-6443, DOI: doi:10.1103/PhysRevA.43.6441.

(90) Svensson, S.; Ausmees, A.; Osborne, S. J.; Bray, G.; Gel'mukhanov, F.; Ågren, H.; Naves de Brito, A.; Sairanen, O.-P.; Kivimäki, A.; Nõmmiste, E.; Aksela, H.; Aksela, S. Observation of an anomalous decay ratio between the molecular field split levels in the S 2p core photoelectron and LVV Auger spectrum of $\mathrm{H}_{2} \mathrm{~S}$. Phys. Rev. Lett. 1994, 72, 3021-3024, DOI: doi:10.1103/PhysRevLett.72.3021.

(91) Thole, B. T.; van der Laan, G. Linear relation between x-ray absorption branching ratio and valence-band spin-orbit expectation value. Phys. Rev. A 1988, 38, 19431947, DOI: doi:10.1103/PhysRevA.38.1943.

(92) Bagus, P. S.; Freund, H.; Kuhlenbeck, H.; Ilton, E. S. A new analysis of X-ray ad- 
sorption branching ratios: Use of Russell-Saunders coupling. Chemical Physics Letters 2008, 455, 331-334, DOI: doi:10.1016/j.cplett.2008.02.085.

(93) Bernini, R. B.; da Silva, L. B. G.; Rodrigues, F. N.; Coutinho, L. H.; Rocha, A. B.; de Souza, G. G. B. Core level (S 2p) excitation and fragmentation of the dimethyl sulfide and dimethyldisulfide molecules. The Journal of Chemical Physics 2012, 136, 144307, DOI: doi:10.1063/1.3701567.

(94) Baba, Y.; Yoshii, K.; Sasaki, T. A. Site-specific fragmentation in condensed $\left(\mathrm{CH}_{3} \mathrm{~S}\right)_{2}$ by sulfur K-edge photoexcitation. The Journal of Chemical Physics 1996, 105, 88588864, DOI: doi:10.1063/1.472615.

(95) Pickering, I. J.; Barney, M.; Cotelesage, J. J. H.; Vogt, L.; Pushie, M. J.; Nissan, A.; Prince, R. C.; George, G. N. Chemical Sensitivity of the Sulfur K-Edge X-ray Absorption Spectra of Organic Disulfides. The Journal of Physical Chemistry A 2016, 120, 7279-7286, DOI: doi:10.1021/acs.jpca.6b06790, PMID: 27571342.

(96) Bergson, G. Molecular orbital treatment of the $3 p \pi$ interaction in five-membered cyclic disulphides. Ark. Kemi 1958, 12, 233.

(97) Bergson, G. A semi-empirical study of the interaction between lone-pair electrons with special reference to the problem of hybridization and the theory of restricted rotation about single bonds. Ark. Kemi 1961, 18, 409.

(98) Wagnière, G.; Hug, W. Polarization and the sign of the long-wavelength cotton-effects in chromophores of symmetry C2. Tetrahedron Letters 1970, 11, 4765-4768, DOI: doi:10.1016/S0040-4039(00)89340-5.

(99) Woody, R. Application of the Bergson model to the optical properties of chiral disulfides. Tetrahedron 1973, 29, 1273-1283, DOI: doi:10.1016/S0040-4020(01)83144-6. 
(100) Carmack, M.; Neubert, L. A. Circular dichroism and the absolute configuration of the chiral disulfide group. Journal of the American Chemical Society 1967, 89, 7134-7136, DOI: doi:10.1021/ja01002a064.

(101) Dodson, R. M.; Nelson, V. C. Correlation of the chirality of the disulfide group with its molecular ellipticity. The Journal of Organic Chemistry 1968, 33, 3966-3968, DOI: doi:10.1021/jo01274a070.

(102) Claeson, G.; Anthoni, U.; Larsen, C.; Nielsen, P. Temperature-Dependent Optical Rotatory Dispersion Measurements of Methyl (R)(-)-1,2-Dithiane-4-carboxylate. The Absolute Configuration of the Disulphide Group. Acta Chemica Scandinavica 1968, 22, 2429-2437.

(103) Ludescher, U.; Schwyzer, R. On the chirality of the cystine disulfide group: Assignment of helical sense in a model compound with a dihedral angle greater than ninety degrees using NMR. and CD. Helvetica Chimica Acta 1971, 54, 1637-1644, DOI: doi:10.1002/hlca.19710540615.

(104) Campbell, J.; Papp, T. Widths of the atomic K-N7 levels. Atomic Data and Nuclear Data Tables 2001, rr7, 1-56, DOI: doi:10.1006/adnd.2000.0848.

(105) Nicolas, C.; Miron, C. Lifetime broadening of core-excited and -ionized states. Journal of Electron Spectroscopy and Related Phenomena 2012, 185, 267-272, DOI: doi:10.1016/j.elspec.2012.05.008, Special Issue in honor of Prof. T. Darrah Thomas: High-Resolution Spectroscopy of Isolated Species. 
Graphical TOC Entry

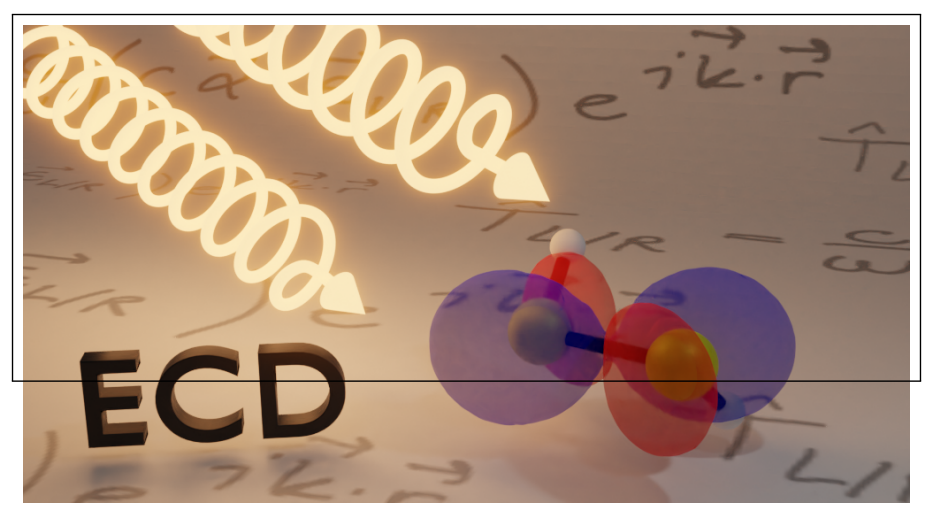




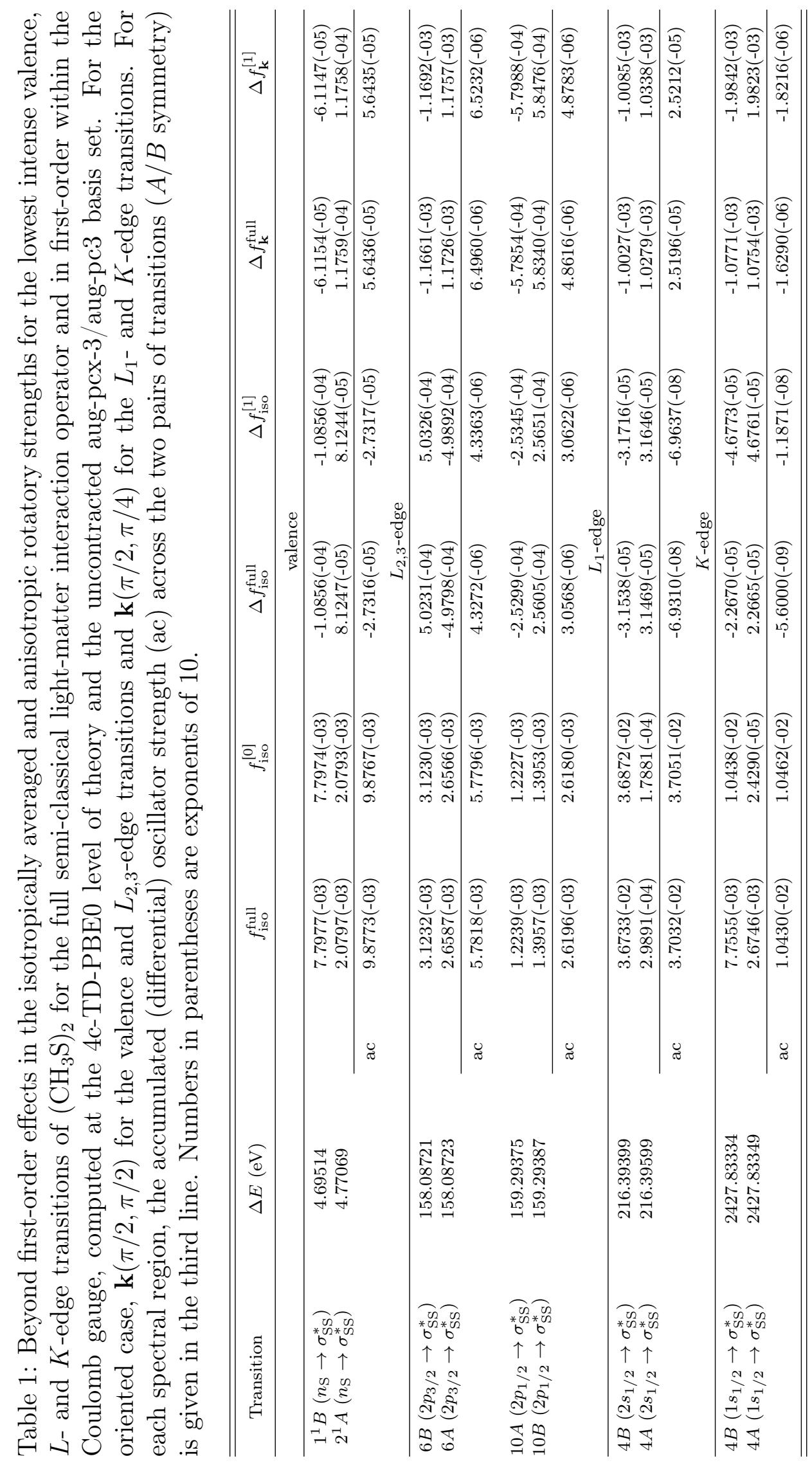


Table 2: Weights of contributions from solid harmonics $((24))$ to the differential cross section $\Delta f(\theta, \phi)$ of selected transitions in $\mathrm{H}_{2} \mathrm{~S}_{2}$. The weights have been scaled by the absolute value of the $s$-contribution. Numbers in parentheses are exponents of 10 .

\begin{tabular}{cl|c|cccc}
\hline \hline Irrep & Excitation & $\Delta f_{\text {iso }}^{[1]}$ & $s$ & $d_{z^{2}}$ & $d_{x^{2}-y^{2}}$ & $d_{x y}$ \\
\hline \multirow{2}{*}{$A$} & Valence & $5.176(-05)$ & 1.000 & -0.447 & 0.300 & -0.056 \\
& $L_{3}$-edge & $-9.118(-05)$ & -1.000 & 0.447 & 0.414 & -0.031 \\
& $L_{2}$-edge & $-2.824(-04)$ & -1.000 & 0.447 & 0.408 & -0.024 \\
& $L_{1}$-edge & $7.316(-05)$ & 1.000 & -0.447 & -0.862 & 8.817 \\
& $K$-edge & $1.657(-04)$ & 1.000 & -0.447 & -0.772 & 9.108 \\
\hline \multirow{2}{*}{$B$} & Valence & $-7.624(-05)$ & -1.000 & -0.328 & -0.090 & 0.314 \\
& $L_{3}$-edge & $9.186(-05)$ & 1.000 & -0.132 & -0.592 & -0.005 \\
& $L_{2}$-edge & $2.883(-04)$ & 1.000 & -0.128 & -0.591 & -0.008 \\
& $L_{1}$-edge & $-7.312(-05)$ & -1.000 & 2.503 & -0.347 & -8.262 \\
& $K$-edge & $-1.657(-04)$ & -1.000 & 0.377 & 0.813 & -9.125 \\
\hline \hline
\end{tabular}

Table 3: Contributions to the isotropic linear and differential oscillator strength $\left(\Delta f_{\text {iso }}^{[2 n+1]}\right.$ and $f_{\text {iso }}^{[2 n]}$ ) at various orders, $n=0,1,2,3$ compared to the result of the full interaction for the two $1 s_{1 / 2} \rightarrow \sigma_{\mathrm{SS}}^{*}$ transitions of $A / B$ symmetry. The errors upon truncation are defined as $\% \delta \Delta f_{\text {iso }}^{[\rightarrow 2 n+1]}=\left|\left(\Delta f_{\text {iso }}^{[\rightarrow 2 n+1]}-\Delta f_{\text {iso }}^{\text {full }}\right) / \Delta f_{\text {iso }}^{\text {full }}\right| \times 100 \%$. The results were obtained using 4c-TD-PBE0 level of theory and the uncontracted aug-pcx-3/aug-pc3 basis set. Numbers in parentheses are exponents of 10 .

\begin{tabular}{ccccc}
\hline \hline & \multicolumn{4}{c}{$4 A\left(1 s_{1 / 2} \rightarrow \sigma_{\mathrm{SS}}^{*}\right)$} \\
\cline { 2 - 5 }$n$ & $f_{\text {iso }}^{[2 n]}$ & $\Delta f_{\text {iso }}^{[2 n+1]}$ & $\% \delta f_{\text {iso }}^{(\rightarrow 2 n)}$ & $\% \delta \Delta f_{\text {iso }}^{[\rightarrow 2 n+1]}$ \\
\hline 0 & $5.5576(-05)$ & $1.6569(-04)$ & 97.99 & 109.10 \\
1 & $3.4276(-03)$ & $-1.0818(-04)$ & 26.07 & 27.42 \\
2 & $-8.1305(-04)$ & $2.4151(-05)$ & 3.36 & 3.06 \\
3 & $9.9900(-05)$ & $-2.2416(-06)$ & $2.57(-1)$ & $2.32(-1)$ \\
4 & $-7.5661(-06)$ & $1.2938(-07)$ & $1.69(-2)$ & $3.96(-1)$ \\
\hline $8(+1)$ & $2.7625(-03)$ & $7.9240(-05)$ & - & - \\
full & $2.7630(-03)$ & $7.9240(-05)$ & - & - \\
\hline \hline & & & & \\
\hline & & $4 B\left(1 s_{1 / 2}\right.$ & $\rightarrow$ & $\left.\sigma_{\text {SS }}^{*}\right)$ \\
\hline 0 & $1.0512(-02)$ & $-1.6570(-04)$ & 35.24 & 109.10 \\
1 & $-3.4601(-03)$ & $1.0818(-04)$ & 9.27 & 27.42 \\
2 & $8.1318(-04)$ & $-2.4153(-05)$ & 1.19 & 3.06 \\
3 & $-9.9897(-05)$ & $2.2413(-06)$ & $9.60(-2)$ & $2.24(-1)$ \\
4 & $7.5513(-06)$ & $-1.2878(-07)$ & $1.43(-3)$ & $3.98(-1)$ \\
\hline $8(+1)$ & $7.7729(-03)$ & $-7.9433(-05)$ & - & - \\
full & $7.7728(-03)$ & $-7.9246(-05)$ & - & - \\
\hline \hline
\end{tabular}

